\title{
Think Beyond the Core : Impact of the Hydrophilic Corona on Drug Solubilization Using Polymer Micelles
}

\section{Haider, Malik Salman}

2020-06-03

Haider, M S , Luebtow, M M , Endres , S, Forster , S , Flegler , V J , Boettcher , B , Aseyev , V , Pöppler , A-C \& Luxenhofer , R 2020 , ' Think Beyond the Core : Impact of the Hydrophilic Corona on Drug Solubilization Using Polymer Micelles ' , ACS Applied Materials \& Interfaces , vol. 12 , no. 22 , pp. 24531-24543 . https://doi.org/10.1021/acsami.9b22495

http://hdl.handle.net/10138/330611

https://doi.org/10.1021/acsami.9b22495

unspecified

acceptedVersion

Downloaded from Helda, University of Helsinki institutional repository.

This is an electronic reprint of the original article.

This reprint may differ from the original in pagination and typographic detail.

Please cite the original version. 
Think Beyond the Core: The Impact of the Hydrophilic Corona on the Drug Solubilization Using Polymer Micelles

Malik Salman Haider ${ }^{1}$, Michael M. Lübtow ${ }^{1}$, Sebastian Endres ${ }^{2}$, Stefan Forster ${ }^{1}$, Vanessa J. Flegler ${ }^{3}$, Bettina Böttcher ${ }^{3}$,Vladimir Aseyev ${ }^{4}$, Ann-Christin Pöppler ${ }^{2}$, Robert Luxenhofer ${ }^{1,4 *}$

${ }^{1}$ Functional Polymer Materials, Chair for Chemical Technology of Material Synthesis and Bavarian Polymer Institute, Faculty of Chemistry and Pharmacy, University of Würzburg, Röntgenring 11, 97070 Würzburg, Germany

${ }^{2}$ Institute of Organic Chemistry, Faculty of Chemistry and Pharmacy, University of Würzburg, Am Hubland, 97074 Würzburg, Germany

${ }^{3}$ Biocenter and Rudolf Virchow Centre, University of Würzburg, Haus D15, Josef-Schneider-Str. 2, 97080 Würzburg, Germany.

${ }^{4}$ Department of Chemistry, University of Helsinki, PB 55, Helsinki FIN-00014, Finland

Correspondence to: robert.luxenhofer@uni-wuerzburg.de; robert.luxenhofer@helsinki.fi

\section{Keywords}

poly(2-oxazoline)s, amphiphilic block copolymers, hydrophobic drugs, nano-formulations, corona-drug interactions,

\section{Abstract}

Polymeric micelles are typically characterized as core-shell structures. The hydrophobic core is considered as depot for hydrophobic molecules and the corona forming block acts as stabilizing and solubilizing interface between core and aqueous milieu. Tremendous efforts have been made to tune the hydrophobic block to increase drug loading and stability of micelles, while the role of hydrophilic blocks is rarely investigated in this context with poly(ethylene glycol) being the gold standard of hydrophilic polymers. To better understand the role of the hydrophilic corona, a small library of structurally similar A-B-A type amphiphiles based on poly(2-oxazoline)s and poly(2-oxazine)s is investigated by varying the hydrophilic block A utilizing poly(2-methyl-2-oxazoline) (pMeOx; A) or poly(2-ethyl-2-oxazoline) (pEtOx; $A^{*}$ ). In terms of hydrophilicity, both polymers closely resemble poly(ethylene glycol). The more hydrophobic block B bear either a poly(2-oxazoline) and poly(2oxazine) backbone with $\mathrm{C} 3$ (propyl) and $\mathrm{C} 4$ (butyl) side chains. Surprisingly, major differences in loading capacities from A-B-A > A*-B-A > A*-B-A* were observed for formulation with the two poorly water-soluble compounds curcumin and paclitaxel, highlighting the importance of the hydrophilic corona of polymer micelles used for drug formulation. The formulations are also characterized by various NMR spectroscopy methods, dynamic light scattering, cryogenic transmission electron microscopy and (micro) differential scanning calorimetry. Our findings suggest that the interaction between the hydrophilic block and the guest molecule should be considered an important but previously largely ignored factor for the rational design of polymeric micelles. 


\section{Introduction}

Amphiphilic block copolymers can self-assemble to form polymeric micelles (PMs), vesicles and many other forms of self-assemblies in selective solvents above their critical micelle concentration $(\mathrm{cmc}){ }^{1}$ Such nanostructures can be employed for a variety of applications such as drug delivery, ${ }^{2}$ viscosity modifications, catalysis and toughening of plastics. ${ }^{3}$ The size, morphology, stability and surface chemistry of PMs can be easily adjusted by fine tuning the hydrophilic/hydrophobic ratios and block lengths. ${ }^{4}$ PMs are capable to encapsulate hydrophobic molecules, increasing their apparent solubility and stability. It is frequently postulated and sometimes confirmed that micelles have core-shell structures, where the core (comprised of the hydrophobic polymer block) hosts the hydrophobic guest molecules, while the shell (comprised of the hydrophilic block) provides the solubility and colloidal stability in aqueous medium. ${ }^{5}$ One early attempt to better understand the interplay of the structure of an polymer amphiphile and a hydrophobic guest/solute is a paper by Kabanov et al. meticulously studying the partitioning of hydrophobic solutes in Pluronic micelles. The micelle formation and hydrophobic partitioning into the micelles are "favored by the hydrophobic interactions in the micelle inner layers, which increase when PPO length increases or PEO length decreases." ${ }^{\prime 6}$ Along these lines, Guo and Lu et al. demonstrated that increasing the chain length of the hydrophobic block has a positive impact on drug loading. ${ }^{7-8}$ However, the interplay between polymer self-assemblies and hydrophobic guests can be quite complex. On the one hand, drug loading can affect the morphologies of self-assemblies, which was shown, among others, by Schulz et al. and Cao et al. ${ }^{4,9}$ On the other hand, Lübtow et al. reported a case where micelle formation of A-B-A triblock copolymers was triggered only by the presence of a hydrophobic guest molecule. ${ }^{10}$ More recently, Wiest et al. showed that increasing the imatinib (an anticancer drug) concentration in taurocholate/lecithin micelles above a critical value, not only causes a morphology transition from vesicles to micelles but also leads to colloidal collapse, i.e. aggregation and precipitation. ${ }^{11}$ PMs have been studied intensively for decades with respect to their physicochemical properties and general behavior in biological systems. ${ }^{12-13}$ However, despite thousands of research papers on the use of PMs for drug delivery, little information can be found on the impact of the hydrophilic block on the drug loading of PMs, the localization of the drug within the micelles ${ }^{4}$ or the colloidal stability ${ }^{14}$ of such drug delivery system.

In the vast majority of described systems for drug delivery the hydrophilic corona is comprised of poly(ethylene glycol) (PEG). ${ }^{15-17}$ However, for several years, alternatives for PEG as the gold standard of a non-fouling, stealth, non-toxic and non-immunogenic synthetic biomaterial have been heavily investigated. ${ }^{18}$ One particularly debated issue is the potential immunogenicity of PEG. ${ }^{19}$ This discussion notwithstanding, alternative polymers ${ }^{20}$ used for the hydrophilic corona of PMs may be useful to tailor the properties of the corona. One such alternative are hydrophilic poly(2-oxazoline)s (POx), in particular poly(2-methyl-2-oxazoline) (pMeOx) and poly(2-ethyl-2-oxazoline) (pEtOx). More recently, poly(2-oxazine)s, their higher main-chain homologues, have also raised some interest. ${ }^{21-25}$ For 
formulation of hydrophobic drugs, ABA type triblock copolymers have been intensively investigated recently and are also in the focus of the present contribution (Scheme 1). In fact, the POx based amphiphiles have demonstrated their unusual drug loading capacity ${ }^{26-29}$ and excellent therapeutic potential in various challenging tumor models. ${ }^{30-36}$ Luxenhofer and coworkers reported an ultra-high paclitaxel (PTX) loaded POx based micellar formulation (loading capacity (LC) $\approx 50 \mathrm{wt} . \%$ $\left(\mathrm{m}_{\text {Drug }} /\left(\mathrm{m}_{\text {Drug }}+\mathrm{m}_{\text {Polymer }}\right)\right)^{37}$ with excellent in vivo anti-tumor efficacy. ${ }^{31-32}$ The lead amphiphile was an AB-A type triblock copolymer, poly(2-methyl-2-oxazoline)-b-(poly(2- $n$-butyl-2-oxazoline)- $b$-poly(2methyl-2-oxazoline) (pMeOx- $b-\mathrm{pBuOx}-b-\mathrm{pMeOx}=\mathrm{A}-\mathrm{pBuOx}-\mathrm{A})$. For variation of the more hydrophobic central B block, Lübtow et al. tested a library of structurally related amphiphiles with a variety of extremely poorly water-soluble molecules like curcumin (CUR), ${ }^{38-40}$ tanshinone IIa and various hydrophobic drugs like efavirenz, dexamethasone and mitotane. ${ }^{41-42}$

a)

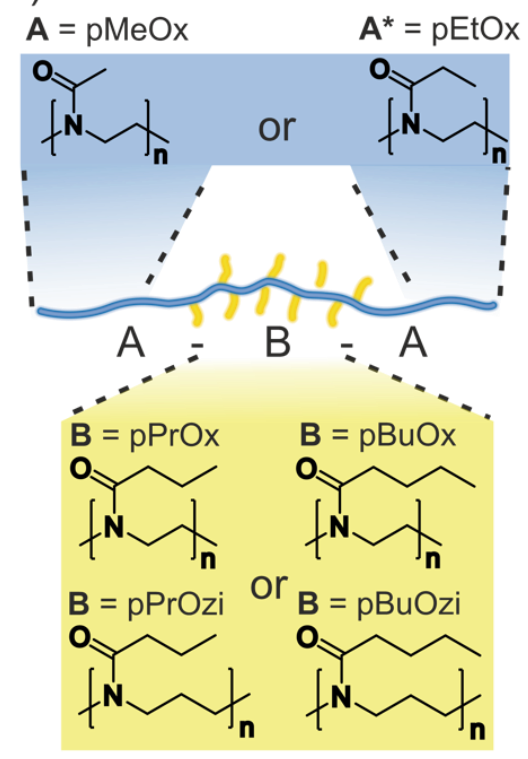

b) Paclitaxel $\mathrm{M}=853.9 \mathrm{~g} / \mathrm{mol}$ $\log P=3.0$

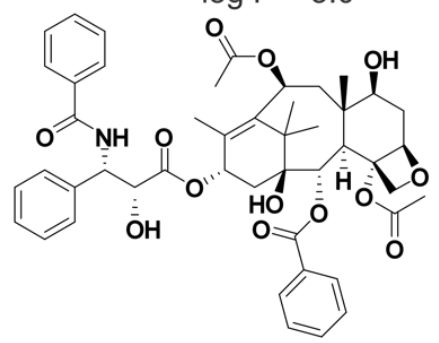

Curcumin $M=369.4 \mathrm{~g} / \mathrm{mol}$

$\log P=3.82$

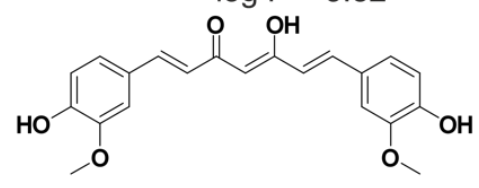

Scheme 1| a) Chemical structures of the A-B-A triblock copolymers used in this study, where hydrophilic blocks (i.e. corona forming blocks) are either poly(2-methyl-2-oxazoline) (pMeOx; A) and/or poly(2-ethyl2-oxazoline (pEtOx, $\mathrm{A}^{*}$ ) and the hydrophobic block (i.e. core forming block) is either pBuOx, pBuOzi, pPrOx or pPrOzi, respectively. Please note, one important feature of these hydrophobic blocks is the presence of highly polar tertiary amide moiety in every repeat unit, rendering the polymer backbone polar also in the hydrophobic block. b) Chemical structure, molecular weight and $\log \mathrm{P}$ value of the model drugs used in this study, curcumin and paclitaxel.

Notably, the small structural difference of only one methylene group switching between polymer backbone and sidechain in A-pBuOx-A and A-pPrOzi-A ((poly(2-methyl-2-oxazoline)- $b$-(poly(2-npropyl-2-oxazine)- $b$-poly(2-methyl-2-oxazoline)) resulted in profound specificities in drug loading. ${ }^{43}$ We and others have spent considerable efforts to elucidate structure-property relationships for different drugs and polymer in this system. However, the preparation and finding of the best polymer/drug 
combinations is very tedious. To accelerate the development of effective drug delivery systems, Alves and coworkers built models predicting LC and loading efficiency (LE) of POx based polymer platform for hydrophobic drugs. ${ }^{44}$

Taking a closer look at the ultra-high loadings ( $\mathrm{LC} \approx 50 \mathrm{wt} \%$ ), which were previously observed for this polymer platform and different drugs including PTX and CUR from a stoichiometric perspective shows how unlikely such high drug loading should be. For example, at the maximum CUR loading of ApPrOzi-A ( $\mathrm{LC} \approx 54$ wt.\%; $12 \mathrm{~g} \mathrm{~L}^{-1}$ CUR for $10 \mathrm{~g} \mathrm{~L}^{-1}$ polymer) 1.3 molecules of CUR $\left(\mathrm{C}_{21} \mathrm{H}_{20} \mathrm{O}_{6}, \mathrm{M}=\right.$ $\left.368 \mathrm{~g} \mathrm{~mol}^{-1}\right)$ for every repeat unit of the hydrophobic block pPrOzi $\left(\mathrm{C}_{7} \mathrm{H}_{13} \mathrm{NO}, \mathrm{M}=127 \mathrm{~g} \mathrm{~mol}^{-1}\right)$ are solubilized in the micelles, assuming essentially all polymer molecules contribute. Therefore, one could hypothesize that not only the hydrophobic repeat units interact with the drug, but also the hydrophilic ones. In fact, very recently we conducted both solid state NMR spectroscopy ${ }^{45}$ [Marvin Grüne, Robert Luxenhofer, Dinu Iuga, Steven P. Brown, Ann-Christin Pöppler*, ${ }^{14} \mathrm{~N}-{ }^{1} \mathrm{H}$ HMQC solid-state NMR Investigation of Amorphous Paclitaxel Formulations, J. Mat. Chem. B, in revision.] as well as small angle neutron scattering experiments ${ }^{46-47}$ which clearly showed the interactions of the hydrophilic corona forming $\mathrm{pMeOx}$ and CUR and its detrimental effect on dissolution rate of lyophilized drug formulations. Also, Cao and co-workers ${ }^{4}$ reported recently in a different polymeric system that at higher drug loading, CUR started to reside in the shell forming block leading to a reduction in the shell hydration. With the ultra-high drug loading and structure-property relationships investigated in detail with respect to the hydrophobic block, ${ }^{43,38}$ we now turn our attention on variations of the hydrophilic block. Accordingly, this work investigates the impact of chemically distinct hydrophilic blocks (pMeOx and pEtOx) on the drug loading (CUR and PTX), solubilization and colloidal stability of POx based ABA triblock copolymers micelles.

\section{Methods and Materials}

All the triblock copolymers were synthesized by living cationic ring opening polymerization $(\mathrm{LCROP})^{48}$ followed by characterization with ${ }^{1} \mathrm{H}-\mathrm{NMR}$ spectroscopy, gel permeation chromatography (GPC), differential scanning calorimetry (DSC), thermogravimetric analysis (TGA), and fluorescence spectroscopy for the determination of critical micelles concentration (CMC). The synthesis of A-pPrOxA, A-pPrOzi-A, A-pBuOx-A and A-pBuOzi-A was reported previously ${ }^{48}, \mathrm{~A}^{*}$-pPrOx-A*, A*-pPrOzi$A^{*}, A^{*}-p B u O x-A^{*}$ and $A^{*}$-pBuOzi-A* triblock copolymers as well as $A^{*}$-pPrOzi-A, A*-pBuOx-A triblock terpolymers were synthesized for this study, synthetic details and full characterization can be found in the supporting information. All the CUR and PTX formulations were prepared by the thin film hydration method. The quantification for drug loading was done with high performance liquid chromatography (HPLC) and UV-Vis spectroscopy. All drug formulations were further characterized by DSC, ${ }^{1} \mathrm{H}-\mathrm{NMR},{ }^{1} \mathrm{H}$ diffusion ordered spectroscopy (DOSY), ${ }^{1} \mathrm{H}-{ }^{1} \mathrm{H}$ nuclear Overhauser effect 
spectroscopy (NOESY), dynamic light scattering (DLS), cryogenic transmission electron microscopy (cryo-TEM) and micro differential scanning calorimetry studies (micro-DSC). The details of each method or procedure are described in the supporting information and referred to in the corresponding sections of the following chapter.

\section{Results and Discussions}

\section{Synthesis and Drug Formulation}

Initially, four structurally similar A-B-A triblock copolymers were synthesized (Table 1 and Scheme 1a) in which we replaced the previously employed $\mathrm{pMeOx}(\mathrm{A})$ with another highly hydrophilic POx, i.e pEtOx $\left(A^{*}\right)$ resulting in $A^{*}$-pPrOx-A*, $A^{*}$-pPrOzi-A*, $A^{*}$-pBuOx-A* and $A^{*}$-pBuOzi-A* triblock copolymer amphiphiles. For the detailed characterization ( ${ }^{1} \mathrm{H}-\mathrm{NMR}, \mathrm{GPC}, \mathrm{TGA}, \mathrm{DSC}$ and fluorescence spectroscopy for cmc determination), the reader is referred to Table 1 and the supporting information (Figures S1-S9, S18-S21, S23, S25).

These four polymers were tested for their solubilizing capacity for PTX and CUR as well-known representatives for poorly water-soluble guest molecules (Figure 1b) by using the thin film method (Figures S26-S30). Briefly, polymer and drug are dissolved in ethanol, which is subsequently removed. The resulting polymer-drug film is dissolved by addition water or other aqueous media. Compared to the polymers bearing a pMeOx hydrophilic block (Figure 1c and Table S1) ${ }^{43}$, a very significant drop in the LC was observed for all A*-B-A* amphiphiles and LCs values never exceeded $30 \mathrm{wt}$ \% (Figure 1d and Table S2). Interestingly, 20-30 wt.\% is typically the upper limit for micellar LC found in literature. ${ }^{49-51}$ It should be noted though, that despite the considerable drop in LC compared to ApPrOzi-A, the LC of A*-pPrOzi-A* based amphiphiles is still much higher than most CUR formulations found in the literature. ${ }^{52}$ For A-pBuOx-A triblock, being the best solubilizer for PTX, ${ }^{31}$ the LC dropped from 48 wt.\% $\left(9 \mathrm{~g} \mathrm{~L}^{-1}\right)$ to $30 \mathrm{wt} . \%\left(4 \mathrm{~g} \mathrm{~L}^{-1}\right)$ for $\mathrm{A}^{*}$-pBuOx-A*. Similarly, for A-pPrOzi-A, being the best solubilizer for CUR, ${ }^{43}$ a drop from $54 \mathrm{wt} . \%\left(11.9 \mathrm{~g} \mathrm{~L}^{-1}\right)$ to $28 \mathrm{wt} . \%\left(3.9 \mathrm{~g} \mathrm{~L}^{-1}\right)$ for $\mathrm{A}^{*}$-pPrOzi-A* was observed. However, both remained the best solubilizers for PTX and CUR, respectively, in the A*B-A* series; i.e. the previously observed drug specificities with respect to the hydrophobic block remained valid. In case of $\mathrm{A}^{*}$-pBuOzi-A*, again a dramatic decrease in LC was observed i.e 48 to 24 wt.\% (9.4 $\mathrm{g} \mathrm{L}^{-1}$ to $\left.3.2 \mathrm{~g} \mathrm{~L}^{-1}\right)$ for CUR and 40 to $16 \mathrm{wt} . \%\left(6.7 \mathrm{~g} / \mathrm{L}\right.$ to $\left.2.1 \mathrm{~g} \mathrm{~L}^{-1}\right)$ for PTX (Table S1 and $\mathrm{S} 2)$. It is important to note, the restricted solubilization capacity cannot be attributed to poor solubility of the newly synthesized A*-B-A* triblock copolymers themselves, as the aqueous solubility of the neat polymers well exceeded $100 \mathrm{~g} \mathrm{~L}^{-1}$. In all the cases with $\mathrm{A} *-\mathrm{B}-\mathrm{A} *$ copolymers, the thin-film method resulted in an undissolved gel-like agglomerate after film-hydration, once a critical LC is reached. ${ }^{1} \mathrm{H}$ $\mathrm{NMR}$ in the non-selective $\mathrm{CDCl}_{3}$ revealed that the agglomerate clearly contains both, the polymer and the drug itself ${ }^{53}$ (Figure S32 and S33). For further characterization, pristine CUR, PTX and freeze-dried 
agglomerate for both PTX and CUR formulations with A*-pBuOx-A*and A*-pPrOzi-A* amphiphiles, respectively, were analyzed using differential scanning calorimetry (DSC).

Table 1| Physico-chemical characterization of all synthesized polymers including the molecular weight $M_{n}$, dispersity $Đ$, glass transition temperature $T_{g}$ and critical micelles concentration $\mathrm{cmc}$ as well as maximum loading capacity (LC) for curcumin (CUR) and paclitaxel (PTX) respectively.

\begin{tabular}{|c|c|c|c|c|c|c|c|c|}
\hline \multirow{3}{*}{ Polymer } & \multirow{3}{*}{$\begin{array}{c}\mathrm{M}_{\mathrm{n}}^{\mathrm{a})} \\
{[\mathrm{kg} / \mathrm{mol}]}\end{array}$} & \multirow{2}{*}{\multicolumn{2}{|c|}{$\begin{array}{c}\mathbf{M}_{\mathbf{n}}{ }^{\mathbf{b})} \\
{[\mathrm{kg} / \mathrm{mol}]}\end{array}$}} & \multirow{3}{*}{$\begin{array}{c}\oplus^{\mathbf{b})} \\
\text { HFIP } \\
\text { DMF }\end{array}$} & \multirow{3}{*}{$\begin{array}{l}\mathbf{T}_{\mathbf{g}}{ }^{\mathbf{c})} \\
{\left[{ }^{\circ} \mathbf{C}\right]}\end{array}$} & \multirow{3}{*}{$\begin{array}{c}\text { cmc }^{\text {d) }} \\
{[\mathrm{mg} / \mathrm{L}]} \\
{[\mathrm{mM}]}\end{array}$} & \multicolumn{2}{|c|}{ max. LC (\%) } \\
\hline & & & & & & & \multirow[b]{2}{*}{ CUR } & \multirow[b]{2}{*}{ PTX } \\
\hline & & HFIP & DMF & & & & & \\
\hline $\mathrm{A}^{*}{ }_{35-} \mathrm{pBuOx}_{20^{-}}$ & 9.5 & 4.1 & 6.6 & 1.11 & 48 & 263 & 18 & 29 \\
\hline $\mathrm{A}^{*}{ }_{35}$ & & & & 1.02 & & 0.027 & & \\
\hline $\mathrm{A}^{*}{ }_{29-} \mathrm{pPrOzi}_{18^{-}}$ & 8.2 & 3.9 & 6.5 & 1.12 & 42 & $\mathbf{\Delta}$ & 28 & 8 \\
\hline$A *_{29}$ & & & & 1.14 & & & & \\
\hline $\mathrm{A}^{*}{ }_{38-\mathrm{pBuOzi}}{ }_{23-}$ & 10 & 3.4 & 6.3 & 1.15 & 37 & 130 & 24 & 16 \\
\hline$A * 38$ & & & & 1.12 & & 0.013 & & \\
\hline \multirow{2}{*}{$\mathrm{A}^{*}{ }_{39}-\mathrm{pPrOx} \mathrm{x}_{29}-\mathrm{A}{ }_{39}$} & 10 & 3.7 & 6.0 & 1.10 & 50 & $\boldsymbol{\Delta}$ & 5 & 1.7 \\
\hline & & & & 1.11 & & & & \\
\hline \multirow[t]{2}{*}{$\mathrm{A}^{*}{ }_{32}-\mathrm{pPrOzi}{ }_{19}-\mathrm{A}_{31}$} & 8.5 & 4.1 & n.d. & 1.11 & 45 & $\boldsymbol{\Delta}$ & 29 & 22 \\
\hline & & & & n.d. & & & & \\
\hline \multirow[t]{2}{*}{$\mathrm{A}_{34-}-\mathrm{pBuOx_{20 } - \mathrm { A } _ { 3 1 }}$} & 8.7 & 3.6 & n.d. & 1.18 & 54 & 485 & 27 & 39 \\
\hline & & & & n.d. & & 0.056 & & \\
\hline \multirow[t]{2}{*}{$\mathrm{pMeOx}_{35}$} & 3.0 & 1.7 & n.d. & 1.09 & 73 & n.d. & 2.2 & n.d. \\
\hline & & & & n.d. & & & & \\
\hline \multirow[t]{2}{*}{$\mathrm{pEtOx}_{35}$} & 3.5 & 1.6 & n.d. & 1.08 & 52 & n.d. & 0.5 & n.d. \\
\hline & & & & n.d. & & & & \\
\hline
\end{tabular}

a) Obtained by ${ }^{1} \mathrm{H}-\mathrm{NMR}$ analysis $\left(\mathrm{CDCl}_{3} ; 300 \mathrm{MHz}\right),{ }^{\text {b) }}$ obtained by $\mathrm{GPC}$ analysis (eluents are HFIP and DMF), ${ }^{\text {c) }}$ obtained by DSC (from $3^{\text {rd }}$ heating curve), ${ }^{\text {d) }}$ obtained by pyrene assay at $25^{\circ} \mathrm{C}$. $\Delta$ Unable to apply the fit to obtain the cmc values.

Clearly, the sharp endothermic peak appearing as the melting point of pristine CUR $\left(175^{\circ} \mathrm{C}\right)$ and solidsolid transition of PTX $\left(170^{\circ} \mathrm{C}\right),{ }^{54}$ were absent in the DSC thermograms of both agglomerates confirming their amorphous character (Figure S34 and S35). To further understand the influence of the hydrophilic corona on drug formulation, two new $\mathrm{A}$-B-A triblock terpolymers with two different hydrophilic blocks were synthesized (for detailed characterization, see Table 1 and Figure S10-S13, S18-S19, S23, S25), selecting the two best performing hydrophobic blocks, pBuOx and pPrOzi (highest LC for PTX and CUR, respectively). The resulting triblock terpolymers are denoted as $\mathrm{A}^{*}$-pBuOx-A and $\mathrm{A}^{*}$-pPrOzi-A. 

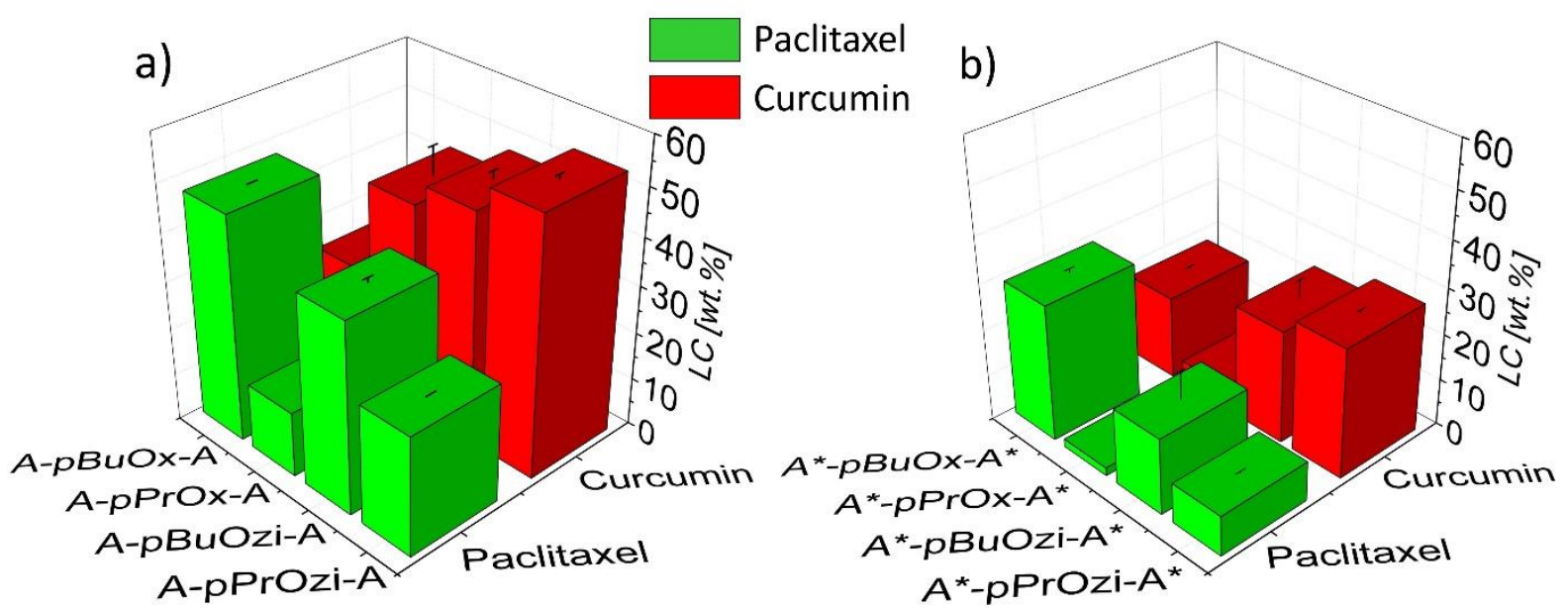

Figure 1. Maximum loading capacity for paclitaxel (green) and curcumin (red) concentrations using four different A-B-A triblock copolymers with a) pMeOx (A) and b) pEtOx ( $\left.\mathrm{A}^{*}\right)$ as hydrophilic blocks (polymer feed $\left.10 \mathrm{~g} \mathrm{~L}^{-1}\right)$. Data is given as means $\pm \mathrm{SD}(\mathrm{n}=3)$. The data shown in $\left.\mathrm{c}\right)$ is taken from reference. ${ }^{43}$ See Table S1-S2 for tabulated values.

Drug formulation experiments revealed that the $\mathrm{LC}$ of $\mathrm{A}$ *-pPrOzi-A and $\mathrm{A} *$-pBuOx-A for CUR (Figure 2a) and PTX (Figure $2 b$ ) fall between A-B-A and $A^{*}-B-A^{*}$, respectively. In the case of CUR, immediate differences could be noted by virtue of the solvatochromicity of CUR. ${ }^{55}$ The formulations with $A^{*}$ pPrOzi-A* and A*-pPrOzi-A appeared orange while A-pPrOzi-A based formulations appeared dark red (Figure 2c) indicating the different microenvironment for the guest molecule. It is clear that CUR solvatochromicity is not only limited to hydrophobic blocks, ${ }^{10,53}$ but also affected by the hydrophilic blocks in these particular micellar formulations. The LC for PTX with A*-pBuOx-A i.e 39 wt.\% (6.5 g $\left.\mathrm{L}^{-1}\right)$ is comparable with A-pBuOx-A i.e 41 wt.\% $\left(7.1 \mathrm{~g} \mathrm{~L}^{-1}\right)$ up to a polymer/PTX feed of 10/8 $\mathrm{g} \mathrm{L}^{-1}$. Upon further increase in drug feed the LC significantly dropped to $3 \mathrm{wt} . \%\left(0.24 \mathrm{~g} \mathrm{~L}^{-1}\right)$, while A-pBuOxA kept solubilizing increasing amounts of PTX (LC $\approx 45$ wt.\%, $8.2 \mathrm{~g} \mathrm{~L}^{-1}$ ) (Figure $2 \mathrm{~b}$ and Table S6). Similarly, for $\mathrm{A}^{*}$-pPrOzi-A/CUR formulations, a dramatic decrease in LC was observed at a polymer/CUR feed $>10 / 6 \mathrm{~g} \mathrm{~L}^{-1}$ (Figure $2 \mathrm{a}$ and Table S5). The formulation completely agglomerated (and sedimented) upon further increase in drug feed. Therefore, the LC for both drugs follows the order of $\mathrm{A}-\mathrm{B}-\mathrm{A}>\mathrm{A}^{*}-\mathrm{B}-\mathrm{A}>\mathrm{A}^{*}-\mathrm{B}-\mathrm{A}^{*}$. Considering the macromolecular structure of the $\mathrm{A}^{*}$-B-A triblock terpolymers, the resulting micelles must inevitable have a mixed pMeOx/pEtOx corona. In contrast, if the corresponding, individual A-B-A and A*-B-A* triblock copolymers are mixed, the system could form mixed micelles or phase separate into two distinct sets of micelles or some kind of intermediate. Accordingly, the two pairs of triblock copolymers were mixed in a 1/1 ratio (w/w), and CUR/PTX formulations were prepared. Interestingly, at first glance, the solubilization results neither resembles the picture expected for mixed micelles or two separate types of micelles. (Figure 2a and b (magenta and cyan bars)). The formulation experiments were also performed for the drugs and the hydrophobic blocks, which are not preferred i.e CUR with pBuOx and PTX with pPrOzi based amphiphiles, respectively (Figure S31, Tables S3 and S4) and a similar picture was obtained. 

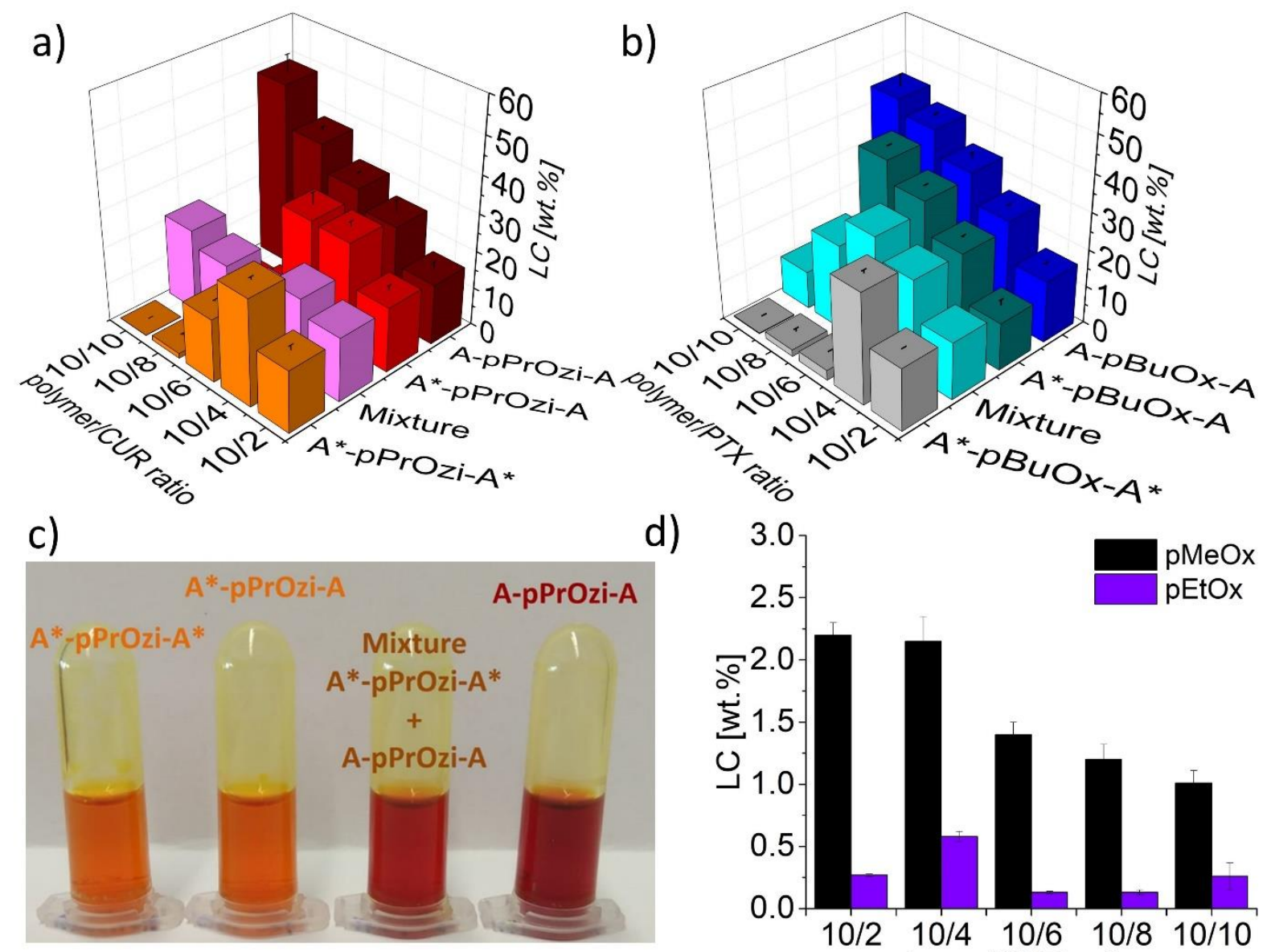

d)

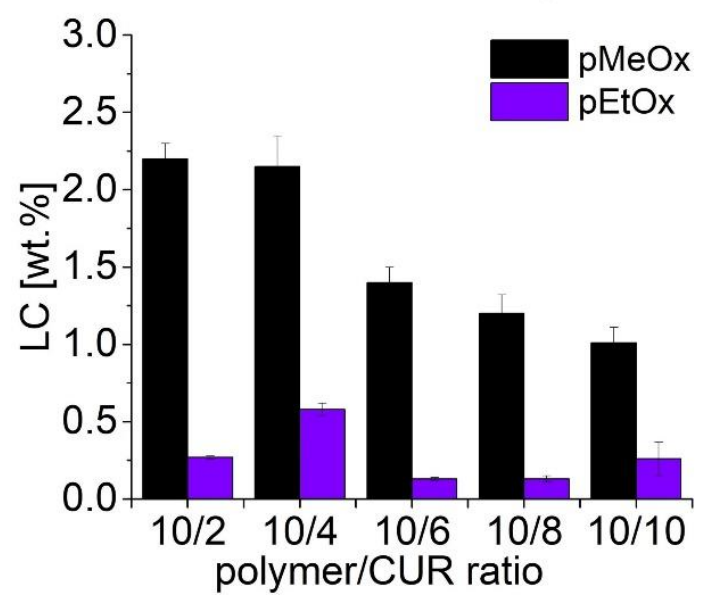

Figure 2| Achieved loading capacity at different ratios (w/w in $g / L$ ) of a) curcumin (CUR) and b) paclitaxel (PTX) and triblock co- and terpolymers with pPrOzi and pBuOx as hydrophobic block and four different setups of hydrophilic blocks i.e A-B-A, A*-B-A, A*-B-A* and a mixture (A-B-A and A*-B-A* 1:1 w/w) $\left(\mathrm{A}=\mathrm{pMeOx}\right.$ and $\left.\left.\mathrm{A}^{*}=\mathrm{pEtOx}\right) . \mathrm{c}\right)$ Visual appearance of CUR aqueous formulation prepared with four different setups of hydrophilic blocks at polymer/CUR feed ratio of 10/2 $\mathrm{g} \mathrm{L}^{-1}$. d) The CUR solubilizing capacity of corona forming blocks as homopolymers pMeOx (black) and pEtOx (violet). In all the cases, the polymer feed was $10 \mathrm{~g} \mathrm{~L}^{-1}$ and the drug feed 0 to $10 \mathrm{~g} \mathrm{~L}^{-1}$. Data is given as means $\pm \mathrm{SD}(\mathrm{n}=3)$. See Tables S5-S7 for tabulated values.

The hydration and thus, water solubility of many water-soluble polymers is strongly temperature dependent and shows a lower critical solution temperature (LCST). While both pMeOx and pEtOx are highly water soluble, pMeOx does not show a LCST in water, ${ }^{56}$ whereas pEtOx can exhibit a LCST depending on the molar mass and polymer architecture with a cloud point temperature $\left(\mathrm{T}_{\mathrm{cp}}\right)$ as low as $61{ }^{\circ} \mathrm{C}$ to $69^{\circ} \mathrm{C} .{ }^{57}$ However, Schubert et al. reported that at least 100 repeat units are required for linear pEtOx to observe the $\mathrm{T}_{\mathrm{cp}}$ below $100^{\circ} \mathrm{C} .^{58}$ During thin-film dissolution, our standard protocol involves heating to $55{ }^{\circ} \mathrm{C}$ and it could be hypothesized that pEtOx undergoes a LCST transition at this temperature due to steric crowding in the micellar corona leading to the observed, low LC values. Therefore, formulation experiments were also performed at room temperature but no significant differences in drug loading were observed (Table S5). To aid with the solubilisation or supersaturation 
of water-insoluble active pharmaceutical ingredients (APIs), also water soluble homopolymers are regularly employed ${ }^{59-60}$ Indeed, $\mathrm{pEtOx}$ has been considered in this context with some success. ${ }^{61}$ Here, pMeOx and pEtOx homopolymers (35 repeat units) were synthesized (Table 1 and Figures S14-S17, S22, S24) and compared for CUR solubilization (Figure $2 d$ and Table S7). It was found that pMeOx is capable to solubilize eight times more CUR $\left(0.23 \mathrm{~g} \mathrm{~L}^{-1}\right)$ than $\mathrm{pEtOx}\left(0.03 \mathrm{~g} \mathrm{~L}^{-1}\right)$, suggesting that pMeOx is a profoundly superior excipient to $\mathrm{pEtOx}$ and also to another commonly employed polymer excipients such as polyvinylpyrrolidone $\left(0.18 \mathrm{~g} \mathrm{~L}^{-1} \text { at } \mathrm{pH} 5.2\right)^{60,62}$ and hydroxypropyl methylcellulose. Interestingly, CUR solvatochromicity was also observed when using the homopolymers pEtOx (orange) and $\mathrm{pMeOx}$ (dark red) for formulation development (Figure 3a). A recent and excellent study by Nischang and co-workers suggested that both $\mathrm{pEtOx}$ and $\mathrm{pMeOx}$ are good alternatives to PEG from the perspective of hydrodynamic invariants. ${ }^{63}$ However, our results highlight important differences between $\mathrm{pMeOx}$ and $\mathrm{pEtOx}$ for pharmaceutical applications which is in line with a recent report by Morgese et al. They compared polymer brushes of PEG, pEtOx, pMeOx as well as mixtures thereof. When compared to $\mathrm{pMeOx}, \mathrm{pEtOx}$ based polymer brushes have shown the least hydration and highest amount of physisorbed proteins. ${ }^{64}$ Likewise, it can be assumed that the profound differences observed in the LC with different hydrophilic blocks are also connected to a difference in hydration between pMeOx and pEtOx and the previously mentioned work by Cao and co-workers ${ }^{4}$ clearly suggests that this effect could be relevant for many other polymers, such as the gold standard poly(ethylene glycol). This would be of greatest relevance for the development of drug delivery systems and a parameter that has been all but ignored previously.

\section{Physicochemical Characterization of Drug Formulations}

\section{Probing Micellar Microenvironment with Reichardt's Dye}

The observed solvatochromicity of CUR in the different micelles shows the presence of different micellar microenvironments. To further probe this, the hydrophobic and solvatochromic Reichardt's dye (RD) was employed to obtain the solvent polarity parameter $\mathrm{E}_{\mathrm{T}}(30),{ }^{65}$ which has been widely used to measure the polarity of different systems ${ }^{66}$ (Figures S36 and S37). RD clearly allowed to distinguish between the polymer sidechain and to a lesser extent polymer backbone in all the investigated samples (polymer/RD 10/0.5 $\mathrm{g} \mathrm{L}^{-1}$ ) (Figure 3b). Albeit somewhat less obvious, the different hydrophilic blocks can also be distinguished. A-pPrOx-A and A-pPrOzi-A showed broad and comparably featureless $\mathrm{UV} / \mathrm{Vis}$ spectra ${ }^{43}$ while $\mathrm{A}^{*}$-pPrOzi-A* and $\mathrm{A}^{*}$-pPrOzi-A featured better resolved local maxima. The latter may be attributed to a more defined molecular environment. A more pronounced difference was observed for polymers featuring a butyl side chain, irrespective of the hydrophilic block. Very clearly distinguishable local maxima between 585 to $595 \mathrm{~nm}$ were observed. Upon co-encapsulating the PTX and RD (polymer/RD/PTX 10/0.5/1 $\mathrm{g} \mathrm{L}^{-1}$ ), all spectra narrowed and aligned with local maxima around $560 \mathrm{~nm}$, indicating the remodeling of the micellar microenvironment (Figure 3c). With an increase in 
PTX feed (from 0 to $6 \mathrm{~g} \mathrm{~L}^{-1}$ ), $\lambda_{\max }$ gradually decreased to 557, 554 and $552 \mathrm{~nm}$ for $\mathrm{A}^{*}$-pBuOx-A*, $\mathrm{A}^{*}$ pBuOx-A and A-pBuOx-A (Figure 3d and Table S9), respectively, with the corresponding $\mathrm{E}_{\mathrm{T}}(30)$ values of 51.3, 51.6 and 51.8. High $\mathrm{E}_{\mathrm{T}}(30)$ values corresponds to high solvent polarity and vice versa (Table S8). Interesting to note, at low PTX loading, the UV-vis spectrum of A-pBuOx-A is clearly separated from $\mathrm{A}^{*}$-pBuOx-A and $\mathrm{A}^{*}$-pBuOx-A*, which are essentially identical up to 9 wt.\% (10/1 g $\left.\mathrm{L}^{-1}\right)$. In contrast, at $23 \mathrm{wt} . \%\left(10 / 3 \mathrm{~g} \mathrm{~L}^{-1}\right)$, the spectra of all three formulations superimpose.
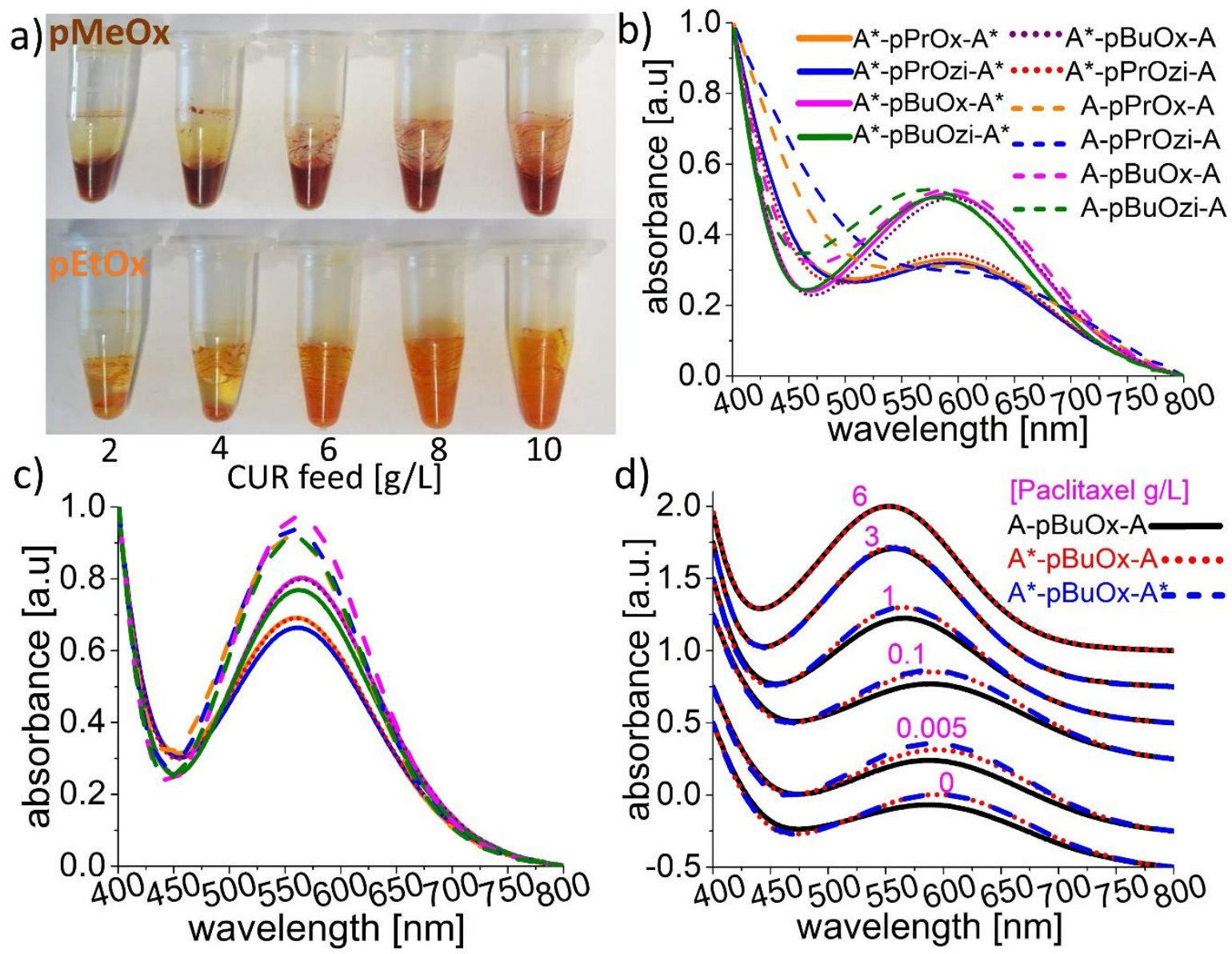

Figure 3| a) Visual appearance of curcumin (CUR) formulations prepared with $\mathrm{pMeOx}$ (upper row) and pEtOx (lower row) homopolymer (polymer feed $10 \mathrm{~g} \mathrm{~L}^{-1}$ and drug feed 0 to $10 \mathrm{~g} \mathrm{~L}^{-1}$ ). Normalized UV-vis absorption spectra of Reichardt's dye in the b) absence (polymer/dye 10/0.5 $\mathrm{g} \mathrm{L}^{-1}$ ) and c) presence of paclitaxel (PTX) (polymer/dye/PTX 10/0.5/1 $\mathrm{g} \mathrm{L}^{-1}$ ). d) Normalized absorption spectra of Reichardt's dye co-formulated with PTX into polymeric micelles of either A-pBuOx-A (black), ${ }^{*}$-pBuOx-A (red) or $\mathrm{A}^{*}$ pBuOx-A* with the increasing PTX feed (0 to $\left.6 \mathrm{~g} \mathrm{~L}^{-1}\right)$ (purple numbers above graph), with the constant polymer/dye feed of $10 / 0.5 \mathrm{~g} \mathrm{~L}^{-1}$. For better visibility, the absorption spectra were shifted along y-axis.

At first sight, this seems to contradict the influence of the polymer corona. However, it should be kept in mind that the microenvironment is probed with RD while increasing PTX feed. The present data can be interpreted that at low loading, the RD molecules can interact with both, the hydrophilic corona and the hydrophobic core. Above a critical loading (9 wt. \% < LC $<23$ wt.\%) the RD only interacts with 
the core, presumably because PTX starts to interact more and preferably with the corona. This observation coincides with the formation of the raspberry-like morphology observed at similar LC values for A-pBuOx-A/PTX formulations, which can be explained in the light of the present work with the start of the interaction of the PTX with the micellar corona. ${ }^{9}$ However, this interpretation should be viewed with considerable care, as we cannot know for sure the location of RD. Unfortunately, a similar analysis using RD is not possible for the CUR formulations, as CUR itself is highly colored and dominates the UV-Vis spectrum over RD.

${ }^{1} H$-NMR Spectroscopy

To gain more insights into the structure of CUR loaded PMs, pure polymers and lyophilized CUR formulations prepared with A-pPrOzi-A, A*-pPrOzi-A, A*-pPrOzi-A* triblocks and a mixture of A-pPrOzi-A/A*-pPrOzi-A* $(1 / 1, w / w)$ were dispersed in $\mathrm{D}_{2} \mathrm{O}$ and subsequently analyzed by ${ }^{1} \mathrm{H}-\mathrm{NMR}$ spectroscopy (Figure 4a, b, Figure 5a, b and Figures S38-S49). Please note, any precipitate that occurred in the formulation was removed and does not contribute to the NMR spectra. Nevertheless, all the data is discussed with respect to the CUR feed, not the actual concentration, which can be found in the supporting information (Table S5). In the absence of CUR, the ${ }^{1} \mathrm{H}-\mathrm{NMR}$ spectra of $\mathrm{A}^{*}$-pPrOzi-A* and $\mathrm{A}^{*}$-pPrOzi-A in $\mathrm{D}_{2} \mathrm{O}$ clearly present all signals from the polymers with the expected intensities (Figure S38 and S39) suggesting that the polymers exist as unimers in a non-aggregated form. The pyrene assay also confirmed the absence of micelles in the case of pPrOzi based amphiphiles (Table 1 and Figure S25). These observations corroborate recent results which showed that A-pPrOzi-A self-assembles only in the presence of hydrophobic molecules. ${ }^{10}$ In the formulations, the characteristic signals for CUR were not observed. ${ }^{10,53}$ 


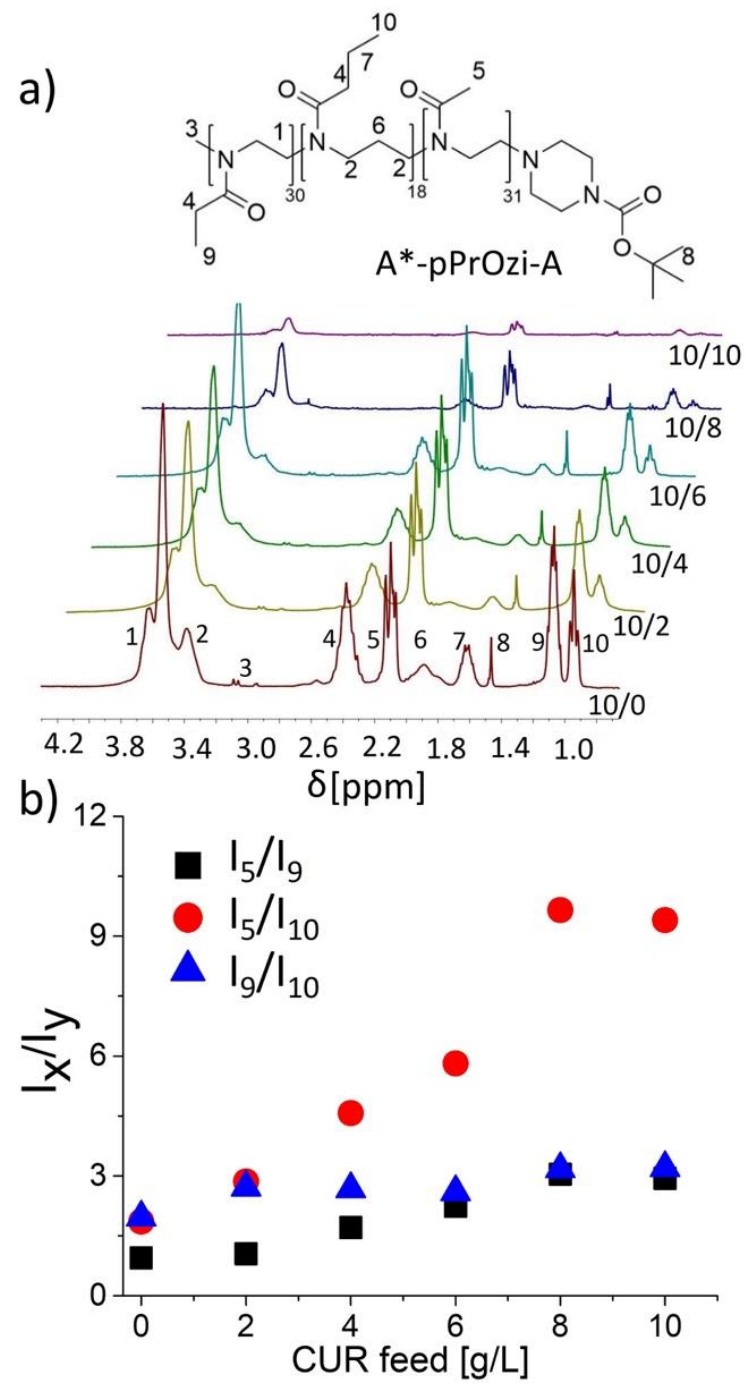

Figure 4| a) ${ }^{1} \mathrm{H}-\mathrm{NMR}$ spectra $\left(\mathrm{D}_{2} \mathrm{O}, 300 \mathrm{MHz}, 298 \mathrm{~K}\right)$ with signal assignment and b) NMR integral ratios $\left(\mathrm{I}_{\mathrm{x}} / \mathrm{I}_{\mathrm{y}}\right)$ of terpolymer (A*-pPrOzi-A)/curcumin (CUR) formulations as a function of CUR feed (0 to $10 \mathrm{~g} \mathrm{\textrm {L } ^ { - }}$ ${ }^{1}$ ). For better visibility, enlarged sections are added as insets.

This can be attributed to a hindered mobility resulting in short $\mathrm{T}_{2}$ relaxation times. In contrast, the polymer signals were clearly distinguishable for all samples, but individual signal intensities strongly varied with CUR loading. ${ }^{67}$ For the A-pPrOzi-A/CUR formulation, comparison of signals attributed to the hydrophilic block vs. the hydrophobic block provides clear evidence for reduced mobility in the latter (Figure S44). However, after a strong initial increase of the integral ratio between $I_{1} / I_{8}$ $\left(\mathrm{CH}_{2}{ }^{\mathrm{MeOx}} / \mathrm{CH}_{3}{ }^{\mathrm{PrOzi}}\right)$ and $\mathrm{I}_{5} / \mathrm{I}_{8}\left(\mathrm{CH}_{3}{ }^{\mathrm{MeOx}} / \mathrm{CH}_{3}{ }^{\mathrm{PrOzi}}\right)$, the ratio decreases again at a CUR feed $\geq 6 \mathrm{~g} \mathrm{~L}^{-1}$, suggesting also a lowered mobility of the hydrophilic block (Figure S45, blue and magenta triangles). This corroborates that the hydrophilic block becomes involved in the coordination of CUR above a critical CUR concentration. This is in line with a recent, more extensive solid-state NMR analysis of A-pPrOzi-A/CUR formulations. ${ }^{45}$ In contrast, for $\mathrm{A}^{*}$-pPrOzi-A*/CUR formulations, the integral ratio $\mathrm{I}_{1} / \mathrm{I}_{9}\left(\mathrm{pEtOx}\right.$ backbone $\left./ \mathrm{CH}_{3}{ }^{\mathrm{PrOzi}}\right)$ and $\mathrm{I}_{8} / \mathrm{I}_{9}\left(\mathrm{CH}_{3}{ }^{\mathrm{EtOx}} / \mathrm{CH}_{3}{ }^{\text {PrOzi }}\right)$ kept on increasing with increasing CUR 
feed (Figure S46 and S47, blue triangle and green diamond) even while overall drug solubilization deteriorated.

a)
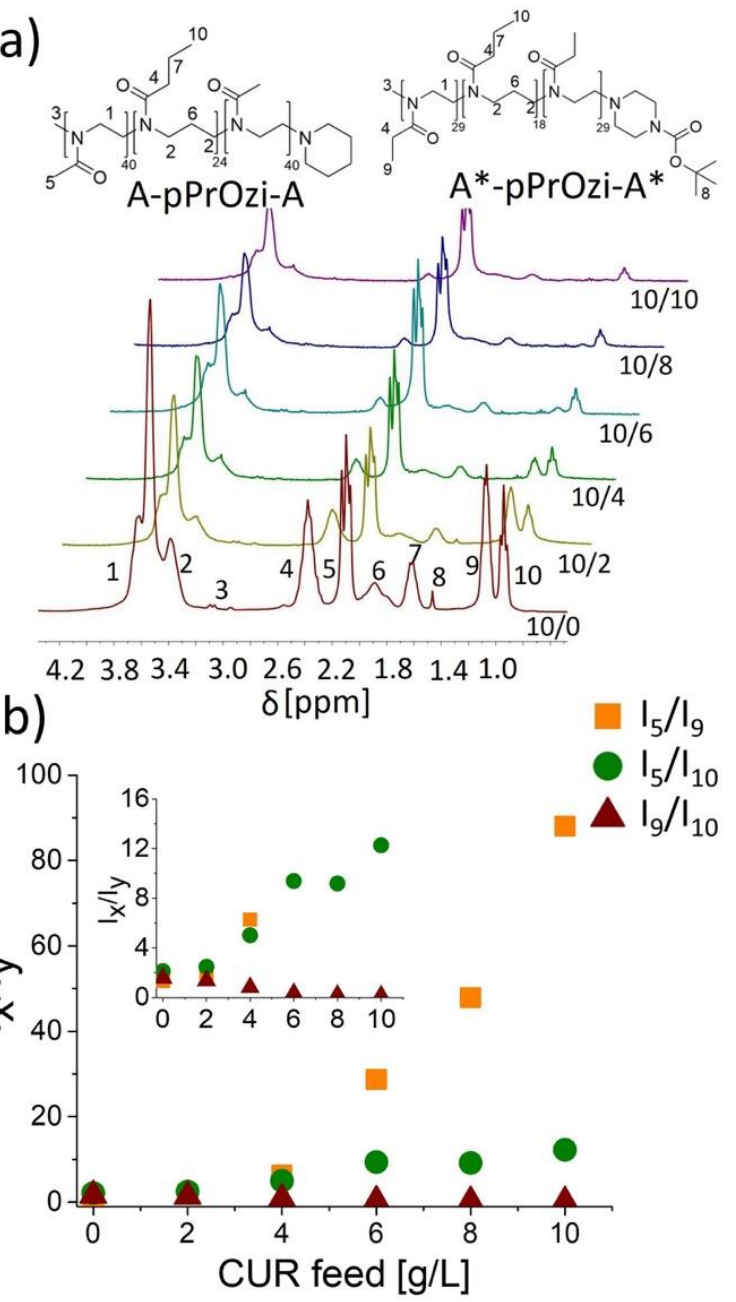

Figure 5| a) ${ }^{1} \mathrm{H}-\mathrm{NMR}$ spectra $\left(\mathrm{D}_{2} \mathrm{O}, 300 \mathrm{MHz}, 298 \mathrm{~K}\right)$ with signal assignment and b) NMR integral ratios $\left(I_{x} / I_{y}\right)$ of mixture (1:1 w/w) A-pPrOzi-A/A*-pPrOzi-A*/curcumin (CUR) formulation as a function of CUR feed $\left(0\right.$ to $\left.10 \mathrm{~g} \mathrm{~L}^{-1}\right)$. For better visibility, enlarged sections are added as insets.

Comparing the spectra of the terpolymer $\mathrm{A}^{*}$-pPrOzi-A formulations (Figure 4) and those employing the mixture $A^{*}$-pPrOzi-A*/A-pPrOzi-A (Figure 5) inform us in more detail on the involvement of different moieties of the hydrophilic coronas and their interactions with CUR.

In case of $A^{*}$-pPrOzi-A/CUR formulation, (Figure $4 a, b$ and Figure S48), the increase in the integral ratio $\mathrm{I}_{5} / \mathrm{I}_{10}\left(\mathrm{CH}_{3}{ }^{\mathrm{MeOx}} / \mathrm{CH}_{3}{ }^{\mathrm{PrOzi}}\right.$, red circles) again indicates the higher mobility of pMeOx compared to pPrOzi with the increasing CUR feed, while the integral ratio $\mathrm{I}_{9} / \mathrm{I}_{10}\left(\mathrm{CH}_{3}{ }^{\mathrm{EtOx}} / \mathrm{CH}_{3}{ }^{\mathrm{PrOzi}}\right.$, blue triangles $)$ did not change significantly (Figure 4b). This suggests that the mobility of EtOx and PrOzi units decreases in a similar manner while $\mathrm{MeOx}$ remains more mobile. More interestingly, in case of the $\mathrm{A}^{*}$ pPrOzi-A*/A-pPrOzi-A mixture, the integral ratio $\mathrm{I}_{5} / \mathrm{I}_{9}\left(\mathrm{CH}_{3}{ }^{\mathrm{MeO}} / \mathrm{CH}_{3}{ }^{\mathrm{EtOx}}\right.$, orange squares) (Figure 5a, $\mathrm{b}$ and Figure S49) increased much more than the ratio $\mathrm{I}_{5} / \mathrm{I}_{10}\left(\mathrm{CH}_{3}{ }^{\mathrm{MeOx}} / \mathrm{CH}_{3}{ }^{\mathrm{PrOzi}}\right.$, green circles)) with 
increasing drug content. The signal of the Boc-protected piperazine terminating reagent (signal 8) attached either at the pMeOx ( $\mathrm{A}^{*}$-pPrOzi-A) or pEtOx $\left(\mathrm{A}^{*}\right.$-pPrOzi-A*) block further corroborates this picture. While the signal remains clearly visible when attached to pMeOx (Figure 4a), it essentially vanishes even at low drug loading when attached to pEtOx (Figure 5a). It appears that a slightly lower hydration of the pEtOx shell compared to $\mathrm{pMeOx}$ is exacerbated with increasing drug loading. The mobility of the hydrophilic pEtOx blocks suffer much more compared to $\mathrm{pMeOx}$ for both terpolymer and the polymer mixture. Quantitatively, however, the increase in the ratio $\mathrm{pMeOx}$ side chain/pEtOx side chain with increasing drug feed was much lower in the case of the triblock terpolymer (Figure 4, black square) vs. the triblock copolymer mixture (Figure 5, orange square), especially as the polymer/drug ratio exceeded 10/4 $\mathrm{g} \mathrm{L}^{-1}$ and was 30 times higher at a 10/10 $\mathrm{g} \mathrm{L}^{-1}$ (Figure 5b, orange squares). The ${ }^{1} \mathrm{H}-\mathrm{NMR}$ analysis of lyophilized supernatant in $\mathrm{CDCl}_{3}$ revealed also the presence of both component of the mixture in the expected ratio up to polymer/CUR feed of 10/4 $\mathrm{g} \mathrm{L}^{-1}$ (Figure S52). Upon further increase in CUR feed, the signal for the EtOx side chain (signal 9) starts to decrease but does not completely vanish even at maximum CUR feed $\left(10 \mathrm{~g} \mathrm{~L}^{-1}\right)$.

This profound loss in pEtOx signal intensity can have two reasons. First, the pEtOx moieties become more solid-like because of strong interaction with CUR and loosing their mobility, probably also segregate more towards the interior of the micelles. Second, the polymers in the mixture could undergo phase separation and micelles that contain more pEtOx, agglomerate/precipitate preferentially, which also let them appear more solid-like but also removes them from the analysis of the supernatant. To elucidate this, the precipitate of $A^{*}$-pPrOzi-A*/A-pPrOzi-A /CUR formulation was analysed (after freeze-drying and dissolution in the non-selective solvent $\mathrm{CDCl}_{3}$ ). Strikingly, the ${ }^{1} \mathrm{H}-\mathrm{NMR}$ analysis revealed exclusively A*-pPrOzi-A* and CUR (Figure S50). This means that the mixture phase separates and $\mathrm{A}^{*}$-pPrOzi-A*/CUR precipitates, while micelles enriched in A-pPrOzi-A remain in solution. In contrast, $\mathrm{pMeOx}$ and $\mathrm{pEtOx}$ are covalently linked in the case of the $\mathrm{A}^{*}$-pPrOzi-A terpolymer and cannot separate. The characteristic signal of the methyl group of $\mathrm{pMeOx}$ in case of the $\mathrm{A}^{*}$-pPrOzi-A/CUR formulation is clearly distinguishable in the ${ }^{1} \mathrm{H}-\mathrm{NMR}$ spectrum (in $\mathrm{CDCl}_{3}$ at $2 \mathrm{ppm}$ ) of the redissolved lyophilized agglomerate (Figure S51). It is obvious that the increasing CUR feed leads to stronger interaction with $A^{*}$-pPrOzi-A* resulting in agglomeration of pure $A^{*}$-pPrOzi-A*/CUR, while the ApPrOzi-A remains in solution and available to solubilize CUR along with a few A*-pPrOzi-A* chains. However, it appears that CUR has a much higher affinity for pEtOx as most of the added CUR is agglomerated with $\mathrm{A}^{*}$-pPrOzi-A*, therefore CUR solubilized by A-pPrOzi-A did not increase with higher feeding ratio (Table S5). In the light of this, the formulation with an excessive CUR feed (polymer/CUR $\left.=10 / 16 \mathrm{~g} \mathrm{~L}^{-1}\right)$ (Table S5) was also prepared expected to saturate A*-pPrOzi-A*. However, no significant increase in solubilized CUR was observed. Again, the ${ }^{1} \mathrm{H}-\mathrm{NMR}$ analysis of the agglomerate (in $\mathrm{CDCl}_{3}$ formed during formulation development showed the massive amount of CUR 
(polymer/CUR $\approx 5 / 12 \mathrm{w} / \mathrm{w}$ ) (Figure S53) further indicating concentration dependent higher interaction of pEtOx with CUR than pMeOx.

Based on these results, we can build a tentative model of the micelle architecture with different hydrophilic coronas and drug loading (Figure 6). At no or very low drug loading, no major difference is observable as the corona-forming polymers are well-hydrated and give excellent colloidal stability for the micelles. However, with increasing drug loading $\mathrm{pEtOx}$ interacts stronger with the hydrophobic guest molecules, compromising its hydration and mobility. Upon further increase of drug loading the $\mathrm{A}^{*}$-B-A* micelles undergo collapse of their hydrophilic corona, and agglomerate/precipitate. In contrast, $\mathrm{A} *$-B-A micelles still have hydrated $\mathrm{pMeOx}$ chains, stabilizing and solubilizing them at somewhat higher drug loading. However, eventually also its capacity is reached and the $\mathrm{A}^{*}$-B-A micelles precipitate earlier than the A-B-A micelles containing only $\mathrm{pMeOx}$, which remain colloidally stable even at ca. $50 \mathrm{wt} . \%$.

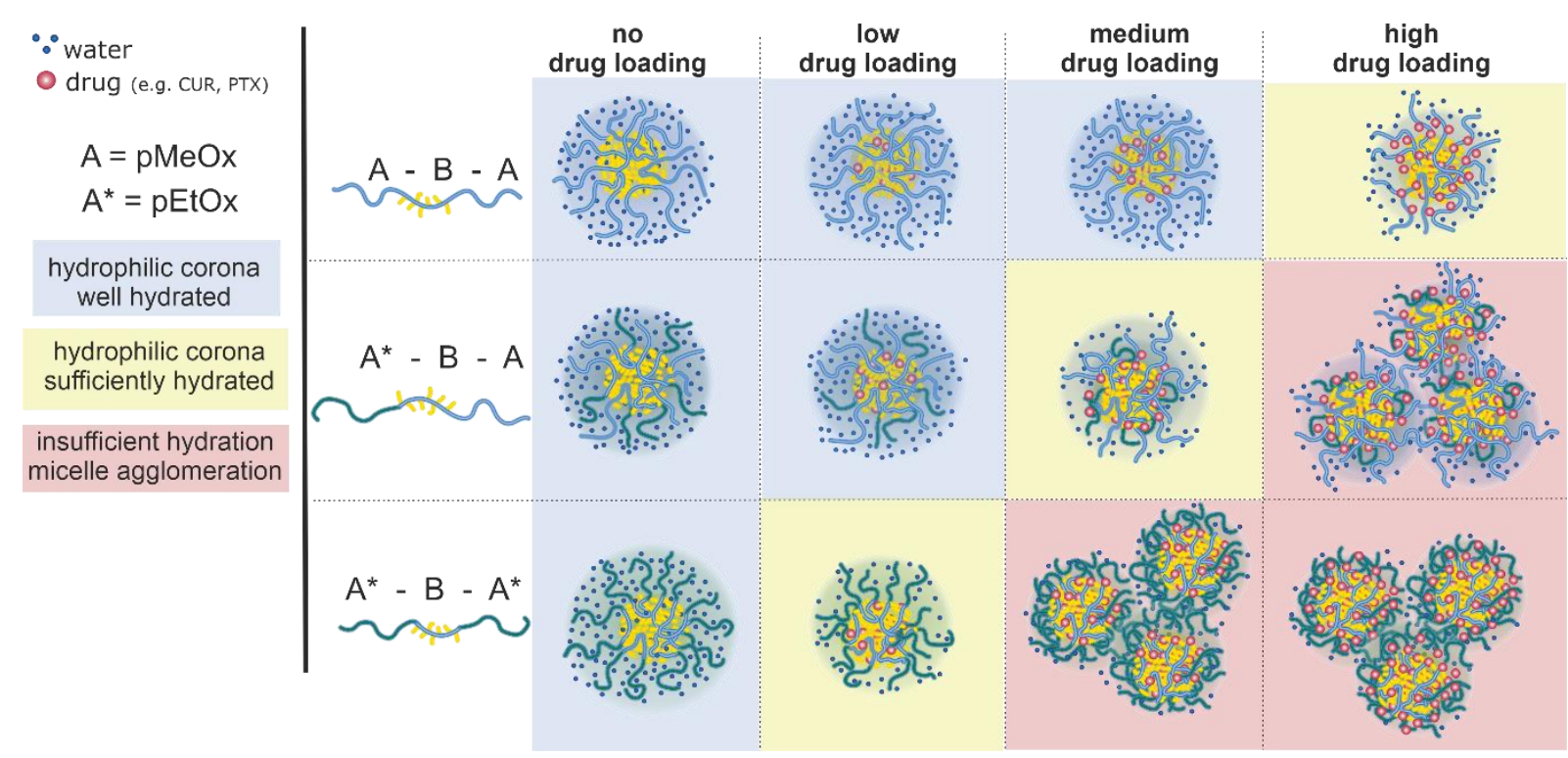

Figure 6| Schematic illustration of the effect of drug loading on the corona of drug loaded micelles according to the corona composition. Without added drug, $\mathrm{pMeOx}$ and $\mathrm{pEtOx}$ coronas are well hydrated. When drug is added, it interacts also with polymer chains comprising the hydrophilic corona, reducing the hydration of the polymer chains. This apparently affects $\mathrm{pEtOx}$ chains more profoundly compared to $\mathrm{pMeOx}$, which is more hydrophilic. While the polymer micelles featuring $\mathrm{pMeOx}$ coronas remain hydrated and colloidally stable even at high drug loading, colloidal stability of pEtOx bearing micelles is compromised.

\section{Diffusion and NOE data}

To support the findings based on comparison of ${ }^{1} \mathrm{H}-\mathrm{NMR}$ and integrations as well as to gain more insights into the assembly of the micelles, ${ }^{1} \mathrm{H}$ diffusion ordered (DOSY) NMR spectroscopy was performed for formulations (polymer/CUR 10/2 $\mathrm{g} \mathrm{L}^{-1}$ ) based on four different setups of hydrophilic 
blocks i.e A-pPrOzi-A, A*-pPrOzi-A, A*-pPrOzi-A* and mixture (A*-pPrOzi-A*/A-pPrOzi-A). Please note, at this CUR feed, solubilization in all formulations was quantitative. From agreement of the diffusion coefficients for water (residual HDO signal) and comparison between a stimulated BPPLED and a double stimulated echo pulse sequence for all four samples, differences in viscosity or convection effects could be excluded. The diffusion coefficients were determined by fitting the experimental diffusion data with a $\log$ normal function ${ }^{68}$ and the values are in comparable range for all four samples indicating similar hydrodynamic radii (Table S10). From comparison of the averaged diffusion coefficients over the functional groups for each sample, the smallest value (and thus largest particles size) was observed for $\mathrm{A}^{*}$-pPrOzi-A* and increases in the order $\mathrm{A}^{*}$-pPrOzi-A $\approx$ polymer mixture $<$ A-pPrOzi-A, which thus shows the smallest hydrodynamic volume. The individual diffusion coefficients for the moieties in the terpolymer and the polymer mixture do not differ in a significant or systematic way. This hints at the presence of mixed micelles for the polymer mixture at 10/2 drug loading. This was further supported by the direct comparison of the respective ${ }^{1} \mathrm{H}$ (Figure S54) and ${ }^{1} \mathrm{H}-$ ${ }^{1} \mathrm{H}$ NOESY NMR spectra (Figure 7a). ${ }^{1} \mathrm{H}-{ }^{1} \mathrm{H}$ NOESY spectra were recorded with different mixing times $\mathrm{d} 8=50$ (Figure S55), 100, 200 and $400 \mathrm{~ms}$ (Figure 7b and c). Each spectrum was carefully phase and baseline corrected before the rows containing the $\mathrm{CH}_{3}{ }^{\text {PrOzi }}$ and $\mathrm{CH}_{3}{ }^{\mathrm{EtOx}}$ groups were extracted to investigate the proximities of these fragments. For the overlay of these 1D extracted slices, the spectra were scaled to equal height of the diagonal peak to compare the relative intensity of the cross-peak. The similarity of the two NOESY spectra (Figure 7a) confirms that, for low CUR loading, the mixture formed mixed micelles. The cross peaks observed in the spectra are largely identical. At strongly increased intensity, cross-peaks between the $\mathrm{CH}_{3}$ groups of EtOx and the PrOzi fragments start to appear. As this cross-peak is very close to the intense diagonal signal, a series of NOESY spectra with increasing mixing time (100, 200 and $400 \mathrm{~ms}$ ) were recorded. For each of these spectra, the rows 1728 and 1684, corresponding to the $\mathrm{CH}_{3}$ groups of PrOzi and EtOx, respectively, were extracted (Figure 7b and c). The $\mathrm{CH}_{3}{ }^{\text {PrOzi }}$ shows indeed a cross-peak with the EtOx signal at $1.02 \mathrm{ppm}$, which increases with increasing mixing time. In fact, this cross-peak is observed for both the terpolymer ( $\mathrm{A}^{*}$-pPrOzi-A) and the mixture. The $\mathrm{CH}_{3}{ }^{\mathrm{EtOx}}$ also exhibits clearly visible cross-peaks to the adjacent $\mathrm{CH}_{2}$ group and to the POx backbone, but also a low intensity signal to the $\mathrm{CH}_{2}{ }^{\text {PrOzi }}$ side chain at $1.57 \mathrm{ppm}$ (Figure 7c). 

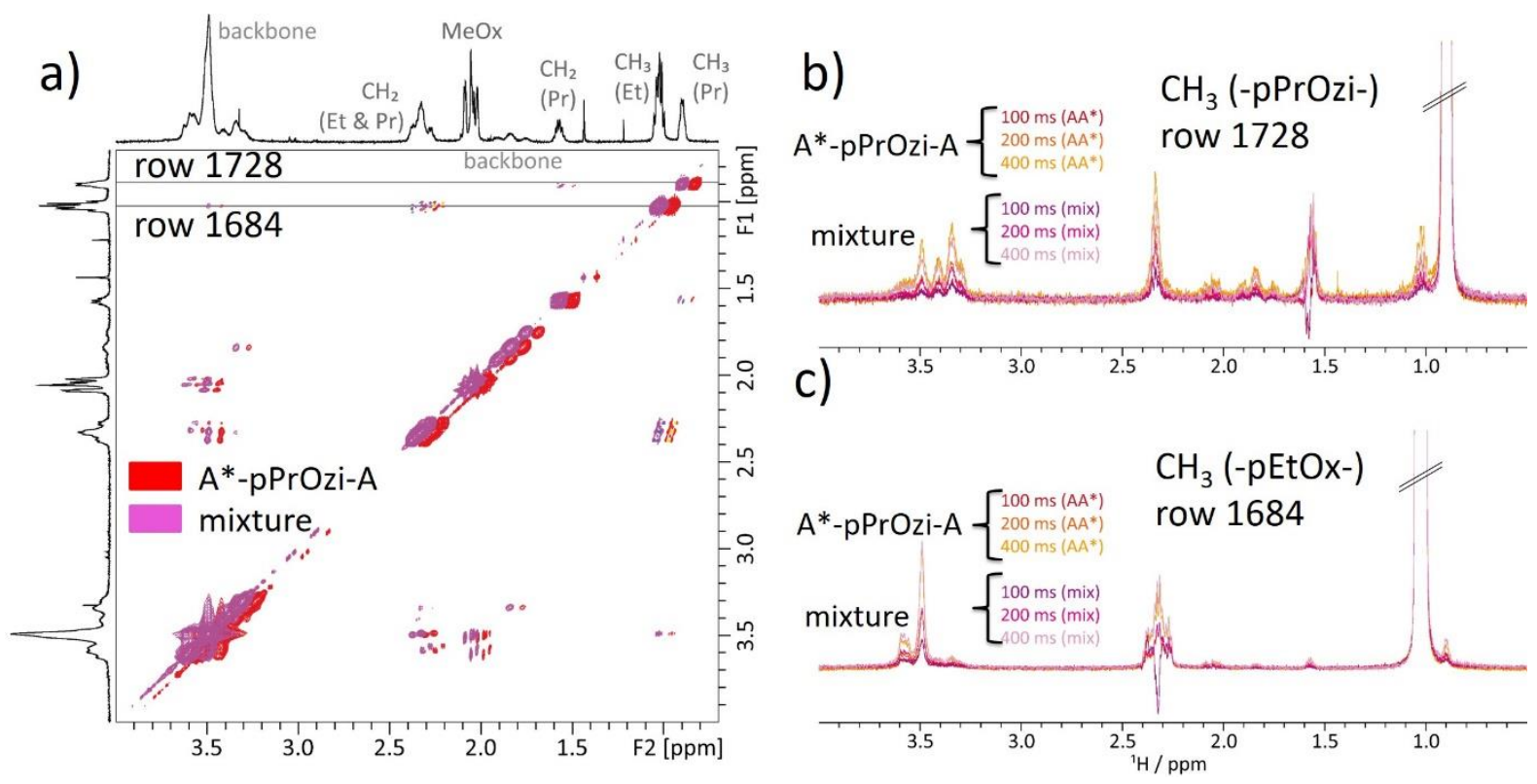

Figure 7| a) Overlay of the ${ }^{1} \mathrm{H}-{ }^{1} \mathrm{H}$ NOESY spectra of curcumin formulation based on $\mathrm{A}^{*}$-pPrOzi-A (terpolymer) (red) and the mixture of A-pPrOzi-A/A*-pPrOzi-A* (magenta) $(1: 1 \mathrm{w} / \mathrm{w})$ with curcumin feed of $2 \mathrm{~g} \mathrm{~L}^{-1}$, recorded with a mixing time of $100 \mathrm{~ms}$. For easier comparison, the red spectrum is slightly shifted to the right. b), c) Overlay of the slices from ${ }^{1} \mathrm{H}-{ }^{1} \mathrm{H}$ NOESY experiments at different mixing times extracted for the two spectral rows as indicated by the grey dashed lines in a).

Interestingly, for both $\mathrm{CH}_{3}$ groups, no contact to $\mathrm{MeOx}$ at $2.05 \mathrm{ppm}$ can be observed at $100 \mathrm{~ms}$ mixing time and only a low intensity signal barely above the noise is detected for the two longer mixing times (200 and $400 \mathrm{~ms}$ ). Presumably this weak, residual contact results from the direct connection of the polymer blocks. All NOESY data strongly suggest that while EtOx units are in close proximity of PrOzi units, the $\mathrm{MeOx}$ units remain more separated. This could be understood as a sign of increased core/corona separation due to the larger hydrophilicity/hydrophobicity contrast.

\section{Dynamic Light Scattering (DLS) and Cryogenic Transmission Electron Microscopy (cryo-TEM)}

To support the results from DOSY NMR spectroscopy, the polymer/CUR $\left(10 / 2 \mathrm{~g} \mathrm{~L}^{-1}\right)$ formulations with four different setups of hydrophilic coronas (A*-pPrOzi-A*, A*-pPrOzi-A, A-pPrOzi-A and mixture of $\mathrm{A}^{*}$-pPrOzi-A*/A-pPrOzi-A) were analyzed by DLS (at $90^{\circ}$ ). All the tested aqueous formulations self-assemble to form micelles with the hydrodynamic radii $\left(\mathrm{R}_{\mathrm{h}}\right)$ ranging between 12 to $15 \mathrm{~nm}$ (Figure S56a). At these subcritical concentrations, the formulations proved to be very stable. After eight months' storage under ambient conditions, no precipitate could be observed by visible inspection and no significant change in the size or size distribution was observed (Figure S56b). Previously, it was observed that A-pBuOx-A/PTX and A-pPrOzi-A/CUR based formulation (with polymer/drug feed of $10 / 2$ and $10 / 3 \mathrm{~g} \mathrm{~L}^{-1}$, respectively) exhibit $\mathrm{R}_{\mathrm{h}}$ of 8 and $11 \mathrm{~nm}$, respectively. ${ }^{9-10} \mathrm{In}$ short, at CUR feed of 2 
$\mathrm{g} \mathrm{L}^{-1}$, where all the employed CUR formulations were colloidally stable, no significant differences in size of the micelles were observed for different hydrophilic coronas. The freshly prepared similar samples were also imaged by cryo-TEM to get better insight into the morphology of the micelles. At first glance, all the four samples (polymer/CUR 10/2 $\mathrm{g} \mathrm{L}^{-1}$ ) (Figure 8a, b, c, d) present as small fairly spherical assemblies with an apparent diameter of 13 to $16 \mathrm{~nm}$. In contrast, cryo-TEM images of ApBuOx-A/PTX formulations that exhibited a more electron-dense core with a less dense, presumable better hydrated pMeOx corona appearing as grey haloes, ${ }^{9}$ the pPrOzi based polymers and CUR formulation appeared as fairly uniform spheres with no clearly discernable internal structure. For comparison, a similar morphology was observed by Bacher et al. for celecoxib loaded casein micelles. ${ }^{69}$ Lübtow et al. found that both at higher $\left(10 / 8 \mathrm{~g} \mathrm{~L}^{-1}\right)$ and lower drug loading $\left(10 / 3 \mathrm{~g} \mathrm{~L}^{-1}\right)$ spherical and worm like structures coexist for A-pPrOzi-A/CUR formulation, while in this case, utilizing a different polymer batch, A-pPrOzi-A/CUR formulation at $10 / 2 \mathrm{~g} \mathrm{~L}^{-1}$ present only as rather spherical assemblies. ${ }^{10}$ It can be concluded that the factors like composition of an amphiphile including hydrophilic and hydrophobic block, chain length of each block, nature and concentration of the $\mathrm{drug}^{4}, 10$ can significantly impact the self-assembly process resulting in defined architectures.
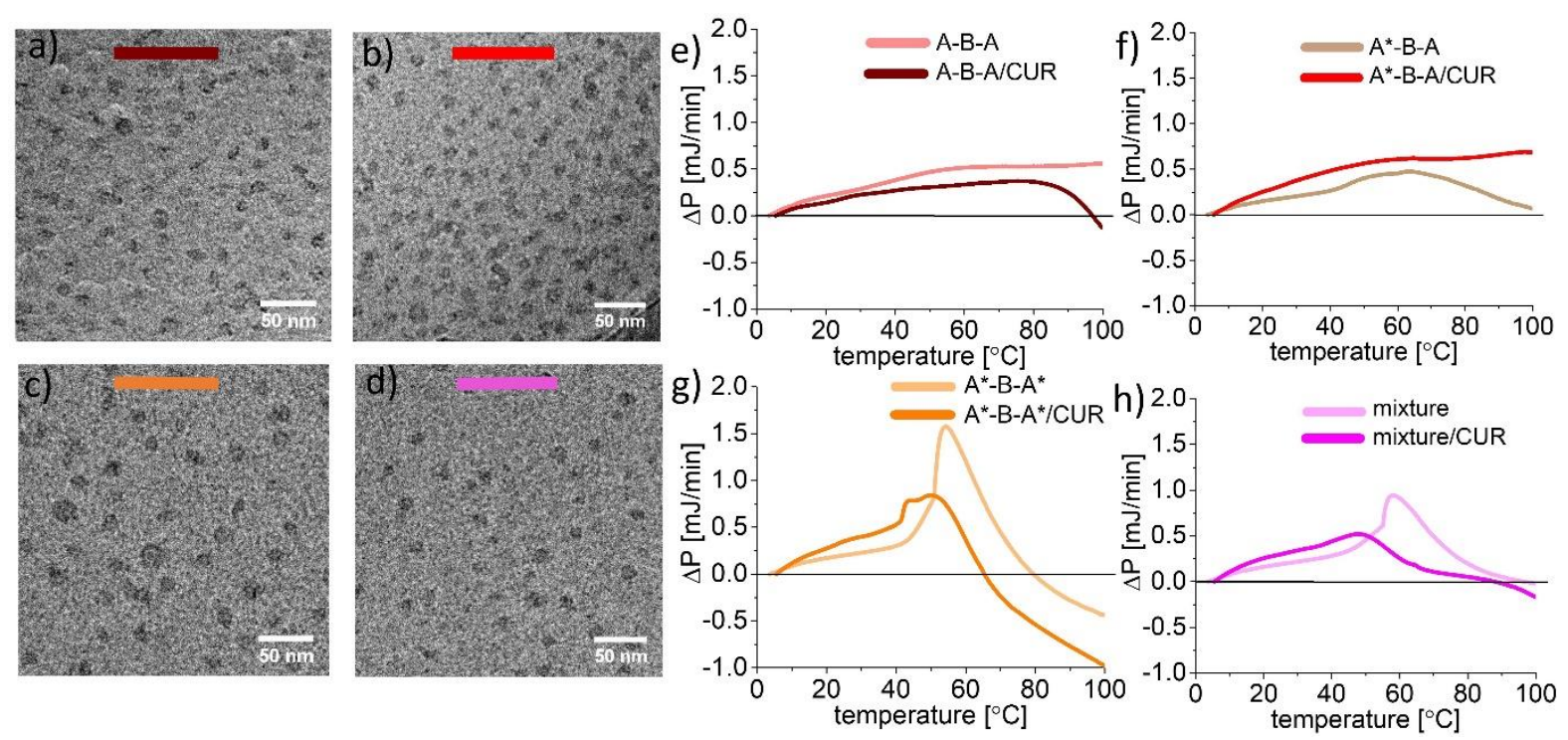

Figure 8| The cryo-TEM images of CUR formulations with four different setups of hydrophilic blocks a) ApPrOzi-A (brown), b) A*-pPrOzi-A (red), c) A*-pPrOzi-A* (orange) and d) mixture (magenta) (A-pPrOzi$\mathrm{A} / \mathrm{A}^{*}$-pPrOzi-A* $\left.1: 1 \mathrm{w} / \mathrm{w}\right)$ at $10 / 2 \mathrm{~g} \mathrm{~L}^{-1}$ polymer/CUR feed $\left(\mathrm{A}^{*}=\mathrm{pEtOx}\right.$ and $\left.\mathrm{A}=\mathrm{pMeOx}\right)$. Raw microcalorimetry heating curves e), f), g) and h) obtained for plain polymers and their respective CUR formulations $\left(10 / 2 \mathrm{~g} \mathrm{~L}^{-1}\right)$ with four different setups of hydrophilic blocks (with heating rate of $60{ }^{\circ} \mathrm{C} / \mathrm{h}$ ). $\Delta \mathrm{P}$ represents the measured heat flow difference, the difference between the sample solution and the solvent reference (DI water) $(\mathrm{n}=1)$.

Micro Calorimetry Studies (micro DSC) 
Differences in hydration of the hydrophilic corona may exacerbate with increasing temperature. Therefore, microcalorimetric studies were conducted for pure polymers with four different setups of hydrophilic corona and their respective CUR formulations (polymer/CUR 10/2 $\mathrm{g} \mathrm{L}^{-1}$ ). Samples were heated from 5 to $100{ }^{\circ} \mathrm{C}$ with a heating rate of $60{ }^{\circ} \mathrm{C} / \mathrm{h}$ and the heat flow difference $(\Delta \mathrm{P})$ between reference (DI water) and sample was recorded (Figure 8e, f, g, h). Clearly, in all cases the pure polymers showed distinct thermograms when compared to their respective CUR formulations. The thermogram of A-B-A plain polymer did not show any evidence of thermal transition up to the measured temperature of $100{ }^{\circ} \mathrm{C}$ (Figure 8e). Interestingly, A-pPrOzi-A/CUR formulations showed a sharp decrease in $\Delta \mathrm{P}$ value above $80{ }^{\circ} \mathrm{C}$, probably due to the interaction of CUR with the pMeOx hydrophilic block in ApPrOzi-A, which decreases pMeOx strength in the interaction with water, leading to a loss of hydration and decrease in $\Delta \mathrm{P}$ value. In the case of $\mathrm{A}^{*}$-pPrOzi-A plain terpolymer (Figure 8f), a thermal transition was observed around $60{ }^{\circ} \mathrm{C}$. In contrast, for the $\mathrm{A}^{*}$-pPrOzi-A/CUR formulation, no evidence of any transition was observed. Besides pMeOx, pEtOx is also present as a hydrophilic block in the $\mathrm{A}^{*}$-pPrOziA terpolymer. Due to the higher affinity of pEtOx to CUR compared to $\mathrm{pMeOx}, \mathrm{pMeOx}$ hydration is not affected at this loading and the system does not go under any thermal transition. This further corroborate the results from NMR experiments showing the preferential condensation and dehydration of the pEtOx over pMeOx blocks upon CUR addition. Most interestingly, the pure A*-pPrOzi-A* polymer showed a sharp thermal transition around $60^{\circ} \mathrm{C}$ with the highest $\Delta \mathrm{P}$ value followed by negative $\triangle \mathrm{P}$ around $80^{\circ} \mathrm{C}$ (Figure $8 \mathrm{~g}$ ). This indicates the dehydration and reorganization of relatively less hydrophilic pEtOx blocks at $60{ }^{\circ} \mathrm{C}$ followed by polymer precipitation around $80{ }^{\circ} \mathrm{C}$. Similarly, $\mathrm{A}^{*}$ pPrOzi-A*/CUR formulations show the most pronounced thermal transition and profound negative $\Delta \mathrm{P}$ values above $60{ }^{\circ} \mathrm{C}$. Notably, A*-pPrOzi-A*/CUR was the only formulation in this series that showed macroscopic agglomeration and precipitation at this loading with increased temperature, which is in line with the strong negative values of $\Delta \mathrm{P}$. Notably, the observed bimodal peak supports the idea that the pEtOx corona dehydrates. Such bimodality maybe attributed to dynamics/kinetics of the transition of the chains in the micellar corona. Such behavior has been reported for core-shell particles and was found to be more pronounced for thermoresponsive polymers of higher molar mass in the corona ${ }^{70}$ or the reorganization of the polymer in corona. It should be noted that the cooling curves of A-pPrOziA/A*-pPrOzi-A*/CUR formulations also showed this bimodality (Figure S57).

Unexpectedly, the thermogram of the A-pPrOzi-A/A*-pPrOzi-A* mixture (Figure 8h) showed close resemblance to the pure $\mathrm{A} *$-pPrOzi-A* polymer (Figure $8 \mathrm{~g}$ ) and entirely different picture from the pure $A^{*}$-pPrOzi-A (Figure 8f), although they comprise the same building blocks. Most interestingly, the mixture of A-pPrOzi-A/A*-pPrOzi-A*/CUR formulation showed an entirely different picture from the terpolymer/CUR formulation. Initially, the obtained curves are similar, but in the temperature range of $36-60{ }^{\circ} \mathrm{C}$ a small but distinct peak is observed in case of the mixture/CUR formulation (Figure $8 \mathrm{~h}$ ). We attribute this thermal transition to the dehydration of predominantly pEtOx chains interacting with 
CUR and concomitant reorganization of the hydrophilic corona. In contrast to the situation with the terpolymer $\mathrm{A}^{*}$-pPrOzi-A formulation, the mixture/CUR formulation can undergo phase separation, which is in fact observed at higher drug loading as previously discussed. At this lower loading, during the measurement, the phase separation is induced by the increase in temperature. In addition, at very high temperature, the microcalorimetric trace of the mixture/CUR formulation resembles the trace for the A-pPrOzi-A/CUR formulation, suggesting also in the mixture, CUR remains to some extent in the corona of A-pPrOzi-A micelles and decrease pMeOx hydration. Again, this corroborates the data obtained by ${ }^{1} \mathrm{H}-\mathrm{NMR}$ for higher loading of CUR.

\section{Summary}

In summary, it is shown conclusively using a range of complementary analytical tools that the hydrophilic corona of polymer micelles plays an important role in solubilization of hydrophobic cargo. Already minor change in the hydrophilic block of a polymer amphiphile (pMeOx vs. pEtOx vs. $\mathrm{pMeOx} / \mathrm{pEtOx}$ ) can have a significant impact on drug loading and/or colloidal stability of the micelles. These findings further suggest that a simple core-shell architecture, where the core incorporate the drug and the corona stabilize the micelle can be an oversimplification. Clearly, the nature of the hydrophobic block and the guest molecule (exemplified here with CUR and PTX) are not the only determinants and more scrutiny must be applied to the interactions between cargo and hydrophilic corona. These interactions can affect system in various ways, which can ultimately modulate the physicochemical properties of nanocarriers. In fact, the highly unusual ultra-high drug loading now well-established for POx based micelles are apparently in part possible due to the choice of the hydrophilic pMeOx. These results shed new light and give clearer instructions on how to design and optimize drug delivery systems and polymeric excipients in general. Considering that the vast majority of drug delivery systems contain poly(ethylene glycol) as a hydrophilic polymer, which is very similar to pEtOx in its amphiphilicity, a question can be raised whether the limited drug loading of polymeric micelles found in the literature, may be strongly correlated to the choice of the hydrophilic polymer. In addition, our finding may also suggest that major differences of the interaction between different hydrophilic polymers and drugs in polymer-drug conjugates or even polymer-protein conjugates can be expected, just as it has been observed for lipopolymers and how they interact with lipid bilayers. ${ }^{71}$

\section{Associated content}

\section{Supporting Information}

Supporting Information is available from the Wiley Online Library or from the corresponding author.

\section{Author Information}

\section{Corresponding Author}


E-mail: robert.luxenhofer@uni-wuerzburg.de

\section{Notes}

M.M.L. and R.L. are listed as inventors on a patent application pertinent to some of the materials discussed in this contribution. R.L. is co-founder and has a financial interest in DelAqua Pharmaceuticals Inc. which is intent on commercializing poly(2-oxazoline) based excipients.

\section{Acknowledgement}

This work was supported by Deutsche Forschungsgemeinschaft (DFG, Project no. 398461692 awarded to R.L). Moreover, M.S.H and M.M.L. are grateful to the Higher education commission of Pakistan and German academic exchange services (HEC-DAAD Pakistan) (M.S. Haider) and Evonik foundation (M.M. Lübtow), respectively for the $\mathrm{PhD}$ scholarships. Financial support from the Fonds der Chemischen Industrie (FCI) in form of a material cost allowance is acknowledged (A.-C. P.). We thank Lukas Hahn and Dr. Matthias Grüne for valuable discussion. 


\section{References}

1. Wang, E.; Lu, J.; Bates, F. S.; Lodge, T. P., Effect of Corona Block Length on the Structure and Chain Exchange Kinetics of Block Copolymer Micelles. Macromolecules 2018, 51, 3563-3571.

2. Garcia Daza, F. A.; Colville, A. J.; Mackie, A. D., Chain architecture and micellization: A mean-field coarse-grained model for poly (ethylene oxide) alkyl ether surfactants. J. Chem. Phys. 2015, 142, 114902-114910.

3. Li, T.; Zhang, J.; Schneiderman, D. K.; Francis, L. F.; Bates, F. S., Toughening glassy poly (lactide) with block copolymer micelles. ACS Macro Lett. 2016, 5, 359-364.

4. Cao, C.; Zhao, J.; Chen, F.; Lu, M.; Khine, Y. Y.; Macmillan, A.; Garvey, C. J.; Stenzel, M. H., Drug-Induced Morphology Transition of Self-Assembled Glycopolymers: Insight into the DrugPolymer Interaction. Chem. Mater. 2018, 30, 5227-5236.

5. Adair, J. H.; Parette, M. P.; Altınoglu, E. I.; Kester, M., Nanoparticulate alternatives for drug delivery. ACS nano 2010, 4, 4967-4970.

6. Kozlov, M. Y.; Melik-Nubarov, N. S.; Batrakova, E. V.; Kabanov, A. V., Relationship between pluronic block copolymer structure, critical micellization concentration and partitioning coefficients of low molecular mass solutes. Macromolecules 2000, 33, 3305-3313.

7. Lu, W.; Li, F.; Mahato, R. I., Poly (ethylene glycol)-block-poly (2-methyl-2-

benzoxycarbonyl-propylene carbonate) micelles for rapamycin delivery: in vitro characterization and biodistribution. J. Pharm. Sci. 2011, 100, 2418-2429.

8. Guo, X. D.; Qian, Y.; Zhang, C. Y.; Nie, S. Y.; Zhang, L. J., Can drug molecules diffuse into the core of micelles? Soft Matter 2012, 8, 9989-9995.

9. $\quad$ Schulz, A.; Jaksch, S.; Schubel, R.; Wegener, E.; Di, Z.; Han, Y.; Meister, A.; Kressler, J.;

Kabanov, A. V.; Luxenhofer, R., Drug-induced morphology switch in drug delivery systems based on poly (2-oxazoline)s. ACS nano 2014, 8, 2686-2696.

10. Lübtow, M. M.; Nelke, L. C.; Seifert, J.; Kühnemundt, J.; Sahay, G.; Dandekar, G.; Nietzer, S. L.; Luxenhofer, R., Drug induced micellization into ultra-high capacity and stable curcumin nanoformulations: Physico-chemical characterization and evaluation in 2D and 3D in vitro models. $J$. Control. Release 2019, 303, 162-180.

11. Wiest, J.; Saedtler, M.; Böttcher, B.; Grüne, M.; Reggane, M.; Galli, B.; Holzgrabe, U.; Meinel, L., Geometrical and structural dynamics of Imatinib within biorelevant colloids. Mol. Pharm. 2018, 15, 4470-4480.

12. Cabral, H.; Matsumoto, Y.; Mizuno, K.; Chen, Q.; Murakami, M.; Kimura, M.; Terada, Y.; Kano, M.; Miyazono, K.; Uesaka, M.; et al, Accumulation of sub-100 nm polymeric micelles in poorly permeable tumours depends on size. Nat. Nanotechnol. 2011, 6, 815-825.

13. Win, K. Y.; Feng, S.-S., Effects of particle size and surface coating on cellular uptake of polymeric nanoparticles for oral delivery of anticancer drugs. Biomaterials 2005, 26, 2713-2722. 
14. Li, Z.; Lenk, T. I.; Yao, L. J.; Bates, F. S.; Lodge, T. P., Maintaining hydrophobic drug supersaturation in a micelle corona reservoir. Macromolecules 2018, 51, 540-551.

15. Gref, R.; Domb, A.; Quellec, P.; Blunk, T.; Müller, R. H.; Verbavatz, J.-M.; Langer, R., The controlled intravenous delivery of drugs using PEG-coated sterically stabilized nanospheres. $A d v$. Drug Deliv. Rev. 1995, 16, 215-233.

16. Diao, Y.-Y.; Li, H.-Y.; Fu, Y.-H.; Han, M.; Hu, Y.-L.; Jiang, H.-L.; Tsutsumi, Y.; Wei, Q.-C.; Chen, D.-W.; Gao, J.-Q., Doxorubicin-loaded PEG-PCL copolymer micelles enhance cytotoxicity and intracellular accumulation of doxorubicin in adriamycin-resistant tumor cells. Int. J. Nanomed. 2011, 6, 1955-1963.

17. Sill, K. N.; Sullivan, B.; Carie, A.; Semple, J. E., Synthesis and Characterization of MicelleForming PEG-Poly (Amino Acid) Copolymers with Iron-Hydroxamate Cross-Linkable Blocks for Encapsulation and Release of Hydrophobic Drugs. Biomacromolecules 2017, 18, 1874-1884.

18. Barz, M.; Luxenhofer, R.; Zentel, R.; Vicent, M. J., Overcoming the PEG-addiction: welldefined alternatives to PEG, from structure-property relationships to better defined therapeutics. Polym. Chem. 2011, 2 (9), 1900.

19. Schellekens, H.; Hennink, W. E.; Brinks, V., The immunogenicity of polyethylene glycol: facts and fiction. Pharm. Res. 2013, 30, 1729-1734.

20. Thomas, A.; Müller, S. S.; Frey, H., Beyond poly (ethylene glycol): linear polyglycerol as a multifunctional polyether for biomedical and pharmaceutical applications. Biomacromolecules 2014, $15,1935-1954$.

21. Bloksma, M. M.; Paulus, R. M.; van Kuringen, H. P. C.; van der Woerdt, F.; LambermontThijs, H. M.; Schubert, U. S.; Hoogenboom, R., Thermoresponsive Poly (2-oxazine) s. Macromol. Rapid Commun. 2012, 33, 92-96.

22. Klein, T.; Parkin, J.; de Jongh, P. A.; Esser, L.; Sepehrizadeh, T.; Zheng, G.; De Veer, M.; Alt, K.; Hagemeyer, C. E.; Haddleton, D. M.; et al, Functional Brush Poly (2-ethyl-2-oxazine) s: Synthesis by CROP and RAFT, Thermoresponsiveness and Grafting onto Iron Oxide Nanoparticles. Macromol. Rapid Commun. 2019, 40, 1800911-1800917.

23. Morgese, G.; Verbraeken, B.; Ramakrishna, S. N.; Gombert, Y.; Cavalli, E.; Rosenboom, J. G.; Zenobi-Wong, M.; Spencer, N. D.; Hoogenboom, R.; Benetti, E. M., Chemical Design of NonIonic Polymer Brushes as Biointerfaces: Poly (2-oxazine) s Outperform Both Poly (2-oxazoline) s and PEG. Angew. Chem. 2018, 57, 11667-11672.

24. Lorson, T.; Jaksch, S.; Lübtow, M. M.; Jüngst, T.; Groll, J.; Lühmann, T.; Luxenhofer, R., A thermogelling supramolecular hydrogel with sponge-like morphology as a cytocompatible bioink. Biomacromolecules 2017, 18, 2161-2171. 
25. Lorson, T.; Lübtow, M. M.; Wegener, E.; Haider, M. S.; Borova, S.; Nahm, D.; Jordan, R.; Sokolski-Papkov, M.; Kabanov, A. V.; Luxenhofer, R., Poly (2-oxazoline) s based biomaterials: A comprehensive and critical update. Biomaterials 2018, 178, 204-280.

26. Lübtow, M. M.; Marciniak, H.; Schmiedel, A.; Roos, M.; Lambert, C.; Luxenhofer, R., Ultrahigh to ultra-low drug loaded micelles: Probing host-guest interactions by fluorescence spectroscopy. Chem. Eur. J. 2019, 25, 12601-12610.

27. Lübtow, M. M.; Oerter, S.; Quader, S.; Jeanclos, E.; Cubukova, A.; Krafft, M.; Haider, M. S.; Schulte, C.; Meier, L.; Rist, M.; et al, In vitro blood-brain-barrier permeability and cytotoxicity of atorvastatin-loaded nanoformulation against glioblastoma in 2D and 3D models. Mol. Pharm. 2020. 28. Salgarella, A. R.; Zahoranová, A.; Šrámková, P.; Majerčíková, M.; Pavlova, E.; Luxenhofer, R.; Kronek, J.; Lacík, I.; Ricotti, L., Investigation of drug release modulation from poly (2-oxazoline) micelles through ultrasound. Sci. Rep. 2018, 8, 9893-9906.

29. Seo, Y.; Schulz, A.; Han, Y.; He, Z.; Bludau, H.; Wan, X.; Tong, J.; Bronich, T. K.; Sokolsky, M.; Luxenhofer, R.; et al, Poly (2-oxazoline) block copolymer based formulations of taxanes: effect of copolymer and drug structure, concentration, and environmental factors. Polym. Adv. Technol. 2015, $26,837-850$.

30. Han, Y.; He, Z.; Schulz, A.; Bronich, T. K.; Jordan, R.; Luxenhofer, R.; Kabanov, A. V., Synergistic combinations of multiple chemotherapeutic agents in high capacity poly (2-oxazoline) micelles. Mol. Pharm. 2012, 9, 2302-2313.

31. Luxenhofer, R.; Schulz, A.; Roques, C.; Li, S.; Bronich, T. K.; Batrakova, E. V.; Jordan, R.; Kabanov, A. V., Doubly amphiphilic poly (2-oxazoline) s as high-capacity delivery systems for hydrophobic drugs. Biomaterials 2010, 31 (18), 4972-4979.

32. He, Z.; Schulz, A.; Wan, X.; Seitz, J.; Bludau, H.; Alakhova, D. Y.; Darr, D. B.; Perou, C. M.; Jordan, R.; Ojima, I., Poly (2-oxazoline) based micelles with high capacity for 3rd generation taxoids: Preparation, in vitro and in vivo evaluation. J. Control. Release 2015, 208, 67-75.

33. He, Z.; Wan, X.; Schulz, A.; Bludau, H.; Dobrovolskaia, M. A.; Stern, S. T.; Montgomery, S. A.; Yuan, H.; Li, Z.; Alakhova, D.; et al, A high capacity polymeric micelle of paclitaxel: Implication of high dose drug therapy to safety and in vivo anti-cancer activity. Biomaterials 2016, 101, 296-309.

34. Paál, K.; Müller, J.; Hegedûs, L., High affinity binding of paclitaxel to human serum albumin. Eur. J. Biochem. 2001, 268, 2187-2191.

35. Wan, X.; Min, Y.; Bludau, H.; Keith, A.; Sheiko, S. S.; Jordan, R.; Wang, A. Z.; Sokolsky Papkov, M.; Kabanov, A. V., Drug Combination Synergy in worm-like Polymeric Micelles Improves Treatment Outcome for Small Cell and Non-Small Cell Lung Cancer. ACS nano 2018, 12, 2426-2439. 36. Zinger, A.; Koren, L.; Adir, O.; Poley, M.; Alyan, M.; Yaari, Z.; Noor, N.; Krinsky, N.; Simon, A.; Gibori, H.; Krayem, M.; et al, Collagenase nanoparticles enhance the penetration of drugs into pancreatic tumors. ACS nano 2019, 13, 11008-11021. 
37. Ma, P.; Mumper, R. J., Paclitaxel nano-delivery systems: a comprehensive review. $J$. Nanomed. Nanotechnol. 2013, 4, 1000164-1000195.

38. Esatbeyoglu, T.; Huebbe, P.; Ernst, I. M.; Chin, D.; Wagner, A. E.; Rimbach, G., Curcuminfrom molecule to biological function. Angew. Chem. 2012, 51, 5308-5332.

39. Naksuriya, O.; Okonogi, S.; Schiffelers, R. M.; Hennink, W. E., Curcumin nanoformulations: a review of pharmaceutical properties and preclinical studies and clinical data related to cancer treatment. Biomaterials 2014, 35, 3365-3383.

40. Baell, J. B., Feeling nature's PAINS: natural products, natural product drugs, and pan assay interference compounds (PAINS). J. Nat. Prod. 2016, 79, 616-628.

41. Lübtow, M. M.; Haider, M. S.; Kirsch, M.; Klisch, S.; Luxenhofer, R., Like Dissolves Like? A Comprehensive Evaluation of Partial Solubility Parameters to Predict Polymer-Drug Compatibility in Ultra-High Drug Loaded Polymer Micelles. Biomacromolecules 2019, 8, 3041-3056.

42. Haider, M. S.; Schreiner, J.; Kendl, S.; Kroiss, M.; Luxenhofer, R., A Micellar Mitotane Formulation with High Drug-Loading and Solubility: Physico-Chemical Characterization and Cytotoxicity Studies in 2D and 3D In Vitro Tumor Models. Macromol. Biosci. 2020, 20, 19001781900191.

43. Lübtow, M. M.; Hahn, L.; Haider, M. S.; Luxenhofer, R., Drug specificity, synergy and antagonism in ultrahigh capacity poly (2-oxazoline)/poly (2-oxazine) based formulations. J. Am. Chem. Soc. 2017, 139, 10980-10983.

44. Alves, V. M.; Hwang, D.; Muratov, E.; Sokolsky-Papkov, M.; Varlamova, E.; Vinod, N.; Lim, C.; Andrade, C. H.; Tropsha, A.; Kabanov, A., Cheminformatics-driven discovery of polymeric micelle formulations for poorly soluble drugs. Sci. Adv. 2019, 5, 9784-9797.

45. Pöppler, A.-C.; Lübtow, M. M.; Schlauersbach, J.; Wiest, J.; Meinel, L.; Luxenhofer, R., Loading-Dependent Structural Model of Polymeric Micelles Encapsulating Curcumin by Solid-State NMR Spectroscopy. Angew. Chem. 2019, 58, 18540-18546.

46. Sochor, B.; Düdükcü, Ö.; Lübtow, M. M.; Schummer, B.; Jaksch, S.; Luxenhofer, R., Probing the Complex Loading Dependent Structural Changes in Ultra-High Drug Loaded Polymer Micelles by Small-Angle Neutron Scattering. Langmuir 2020, 36, 3494-3503.

47. Geisel, K.; Rudov, A. A.; Potemkin, I. I.; Richtering, W., Hollow and core-shell microgels at oil-water interfaces: Spreading of soft particles reduces the compressibility of the monolayer.

Langmuir 2015, 31, 13145-13154.

48. Witte, H.; Seeliger, W., Cyclische imidsäureester aus nitrilen und aminoalkoholen. Leibigs Ann. 1974, 1974, 996-1009.

49. Yu, Y.; Chen, C.-K.; Law, W.-C.; Weinheimer, E.; Sengupta, S.; Prasad, P. N.; Cheng, C., Polylactide-graft-doxorubicin nanoparticles with precisely controlled drug loading for $\mathrm{pH}$-triggered drug delivery. Biomacromolecules 2014, 15, 524-532. 
50. Cheetham, A. G.; Zhang, P.; Lin, Y.-a.; Lock, L. L.; Cui, H., Supramolecular nanostructures formed by anticancer drug assembly. J. Am. Chem. Soc. 2013, 135, 2907-2910.

51. Maksimenko, A.; Dosio, F.; Mougin, J.; Ferrero, A.; Wack, S.; Reddy, L. H.; Weyn, A.-A.; Lepeltier, E.; Bourgaux, C.; Stella, B., A unique squalenoylated and nonpegylated doxorubicin nanomedicine with systemic long-circulating properties and anticancer activity. Proc. Natl. Acad. Sci. U.S.A 2014, 111, 217-226.

52. Raveendran, R.; Mullen, K. M.; Wellard, R. M.; Sharma, C. P.; Hoogenboom, R.; Dargaville, T. R., Poly (2-oxazoline) block copolymer nanoparticles for curcumin loading and delivery to cancer cells. Eur. Polym. J. 2017, 93, 682-694.

53. Lübtow, M. M.; Keßler, L.; Appelt Menzel, A.; Lorson, T.; Gangloff, N.; Kirsch, M.; Dahms, S.; Luxenhofer, R., More Is Sometimes Less: Curcumin and Paclitaxel Formulations Using Poly (2oxazoline) and Poly (2-oxazine)-Based Amphiphiles Bearing Linear and Branched C9 Side Chains. Macromol. Biosci 2018, 18, 1800155-1800172.

54. Liggins, R. T.; Hunter, W.; Burt, H. M., Solid-state characterization of paclitaxel. J. Pharm. Sci. 1997, 86, 1458-1463.

55. Patra, D.; Barakat, C., Synchronous fluorescence spectroscopic study of solvatochromic curcumin dye. Spectrochim. Acta A 2011, 79, 1034-1041.

56. Hoogenboom, R.; Schlaad, H., Thermoresponsive poly (2-oxazoline) s, polypeptoids, and polypeptides. Polym. Chem. 2017, 8, 24-41.

57. Lin, P.; Clash, C.; Pearce, E. M.; Kwei, T.; Aponte, M., Solubility and miscibility of poly (ethyl oxazoline). J. Polym. Sci. B 1988, 26, 603-619.

58. Hoogenboom, R.; Thijs, H. M.; Jochems, M. J.; van Lankvelt, B. M.; Fijten, M. W.; Schubert, U. S., Tuning the LCST of poly (2-oxazoline) s by varying composition and molecular weight: alternatives to poly (N-isopropylacrylamide)? ChemComm 2008, 44, 5758-5760.

59. Li, B.; Konecke, S.; Wegiel, L. A.; Taylor, L. S.; Edgar, K. J., Both solubility and chemical stability of curcumin are enhanced by solid dispersion in cellulose derivative matrices. Carbohydr. Polym. 2013, 98, 1108-1116.

60. Paradkar, A.; Ambike, A. A.; Jadhav, B. K.; Mahadik, K., Characterization of curcumin-PVP solid dispersion obtained by spray drying. Int. J. Pharm. 2004, 271, 281-286.

61. Bender, J. C. M. E.; Hoogenboom, R.; Van Vliet, P. A. A. Drug delivery system comprising polyoxazoline and a bioactive agent. 2014.

62. Hernandez-Patlan, D.; Solis-Cruz, B.; Pontin, K. P.; Latorre, J. D.; Baxter, M. F.; HernandezVelasco, X.; Merino-Guzman, R.; Méndez-Albores, A.; Hargis, B. M.; Lopez-Arellano, R., Evaluation of a solid dispersion of curcumin with polyvinylpyrrolidone and boric acid against Salmonella Enteritidis infection and intestinal permeability in broiler chickens: A pilot study. Front. Microbiol. 2018, 9, 1289-1299. 
63. Grube, M.; Leiske, M. N.; Schubert, U. S.; Nischang, I., POx as an alternative to PEG? A hydrodynamic and light scattering study. Macromolecules 2018, 51, 1905-1916.

64. Morgese, G.; Gombert, Y.; Ramakrishna, S. N.; Benetti, E. M., Mixing Poly (ethylene glycol) and Poly (2-alkyl-2-oxazoline) s Enhances Hydration and Viscoelasticity of Polymer Brushes and Determines Their Nanotribological and Antifouling Properties. ACS Appl. Mater. Interfaces 2018, 10, 41839-41848.

65. Reichardt, C., Solvatochromic dyes as solvent polarity indicators. Chem. Rev. 1994, 94, 23192358 .

66. Cerón-Carrasco, J. P.; Jacquemin, D.; Laurence, C.; Planchat, A.; Reichardt, C.; Sraïdi, K., Solvent polarity scales: determination of new ET (30) values for 84 organic solvents. J. Phys. Org. Chem. 2014, 27, 512-518.

67. de Graaf, A. J.; Boere, K. W.; Kemmink, J.; Fokkink, R. G.; van Nostrum, C. F.; Rijkers, D. T.; van der Gucht, J.; Wienk, H.; Baldus, M.; Mastrobattista, E., Looped structure of flowerlike micelles revealed by 1H NMR relaxometry and light scattering. Langmuir 2011, 27, 9843-9848. 68. Guo, X.; Laryea, E.; Wilhelm, M.; Luy, B.; Nirschl, H.; Guthausen, G., Diffusion in Polymer Solutions: Molecular Weight Distribution by PFG-NMR and Relation to SEC. Macromol. Chem. Phys. 2017, 218, 1600440-1600450.

69. Bachar, M.; Mandelbaum, A.; Portnaya, I.; Perlstein, H.; Even-Chen, S.; Barenholz, Y.; Danino, D., Development and characterization of a novel drug nanocarrier for oral delivery, based on self-assembled $\beta$-casein micelles. J. Control. Release 2012, 160, 164-171.

70. Shan, J.; Chen, J.; Nuopponen, M.; Tenhu, H., Two phase transitions of poly (Nisopropylacrylamide) brushes bound to gold nanoparticles. Langmuir 2004, 20, 4671-4676.

71. Magarkar, A.; Róg, T.; Bunker, A., A computational study suggests that replacing PEG with PMOZ may increase exposure of hydrophobic targeting moiety. Eur. J. Pharm. Sci. 2017, 103, 128135 . 